\title{
AUTOMATIC REES MATRIX SEMIGROUPS OVER CATEGORIES
}

\author{
Mark Kambites \\ Fachbereich Mathematik / Informatik, Universität Kassel \\ 34109 Kassel, Germany \\ kambites@theory.informatik.uni-kassel.de
}

\begin{abstract}
We consider the preservation of the properties of automaticity and prefix-automaticity in Rees matrix semigroups over semigroupoids and small categories. Some of our results are new or improve upon existing results in the single-object case of Rees matrix semigroups over semigroups.
\end{abstract}

\section{INTRODUCTION}

In recent years, one of the most productive areas of combinatorial group theory has been the theory of automatic groups. The expression of a finitely generated group using an automatic structure provides a basis for efficiently performing computations in the group which may be hard or impossible given only a presentation. Groups which admit automatic structures also display a number of remarkable algebraic and geometric properties. [7]

The usual language-theoretic definition of automaticity in groups lends itself naturally to application in wider contexts. It is observed in [7] that the notion of automaticity extend naturally from groups to groupoids. More recently, Hudson [15] has introduced a definition of automaticity in semigroups and monoids and a theory of automatic semigroups has begun to emerge [2, 3, 4, 5, 6, 9, 10, 11, 12, 13. In [17, we developed a common framework for these generalisations, in the form of a theory of automaticity for small categories and semigroupoids. We explored what could be learnt about automatic small categories and semigroupoids, by applying the existing theory of automatic semigroups. Semigroupoids and small categories play a vital role in the structural theory of semigroups, so it also seems natural to ask, conversely, whether automatic categories can tell us anything about automatic semigroups.

A key recurring theme in structural semigroup theory is that of the Rees matrix construction (see, for example, 14]). A number of interesting results have been proved concerning the relationship between automaticity properties and Rees matrix constructions over groups [4] and more generally over semigroups [5, 21]. At the same time, Lawson [19] has applied to the study of abundant semigroups a form of Rees matrix construction over

2000 Mathematics Subject Classification. 20M05, 20M50, 18 B40.

Key words and phrases. Rees matrix semigroups, categories, automatic structures. 
semigroupoids. In [18 we explored combinatorial aspects of this construction showing that, under certain assumptions, combinatorial properties such as finite generation and finite presentability are preserved. In this paper, we consider the relationship between automaticity and these constructions. Some of our results are new, or improve upon existing results, even when specialised to the single-object case of Rees matrix semigroups over semigroups.

In addition to this introduction, this paper comprises four sections. In Section 2 we briefly recall the notions of generators and relations for partial algebras which we studied in [18, and the definitions of regular path languages and of automatic and prefix-automaticity introduced in [17. We also recall some key results from [17] which will be applied in this paper. In Section 3 , we prove some technical results concerning automaticity in small categories and semigroupoids, which will be needed in the sections that follow.

Section 4 considers the relationship between automaticity and Rees matrix constructions with zero over semigroupoids. We show that a finitely generated Rees matrix semigroup over an automatic semigroupoid is always automatic, and provide some sufficient conditions for the underlying semigroupoid of an automatic Rees matrix semigroup to be automatic. In

Section [5] we prove some related results for prefix-automaticity. Section [6] extends these results to the case of Rees matrix constructions without zero. Finally, Section 7 contains some remarks and open questions.

\section{BACKGROUND}

In this section, we briefly recall a number of definitions and results from [18] and [17. For a more detailed introduction, the reader should consult those papers.

By a (directed) graph $X$ we mean a collection $X^{0}$ of vertices together with a collection $X^{1}$ of edges and two functions $\alpha, \omega: X^{1} \rightarrow X^{0}$ which determine respectively the source and target of each edge.

A path $\pi$ in $X$ is a finite sequence $e_{1} e_{2} \ldots e_{n}$ of (not necessarily distinct) edges in $X$ such that $e_{i} \omega=e_{i+1} \alpha$ for $1 \leq i<n$. We define $\pi \alpha=e_{1} \alpha$, $\pi \omega=e_{n} \omega$, and call these the source and target respectively of the path $\pi$. The length of the path $\pi$ is the number $n$ of edges; we denote it by $|\pi|$. There is also a distinct empty path of length 0 at each vertex, with source and target that vertex. We identify each path of length 1 with its single edge, and each vertex with the empty path at that vertex. Thus, $X^{0}$ and $X^{1}$ are the sets of paths in $X$ of length 0 and of length 1 respectively. We extend this notation by writing $X^{n}$ for the set of paths of length $n$ in $X$. We also write $X^{\geq n}$ for the set of paths in $X^{*}$ of length $n$ or more; $X^{>n}$, $X^{\leq n}$ and $X^{<n}$ are defined analogously in the obvious way, including empty paths where appropriate.

Let $X$ and $Y$ be graphs. The direct product $X \times Y$ of $X$ and $Y$ is the (finite) graph with vertex set $X^{0} \times Y^{0}$, and edge set $X^{1} \times Y^{1}(f, g) \alpha=$ $(f \alpha, g \alpha)$ and $(f, g) \omega=(f \omega, g \omega)$ for all $f \in X^{1}, g \in Y^{1}$. A graph morphism $\sigma: X \rightarrow Y$ consists of functions $\sigma^{0}: X^{0} \rightarrow Y^{0}$ and $\sigma^{1}: X^{1} \rightarrow Y^{1}$ such that $x \sigma^{1} \alpha=x \omega \alpha^{0}$ and $x \sigma^{1} \omega=x \omega \sigma^{0}$ for all edges $x \in X^{1}$. If, in addition, 
$X^{0}=Y^{0}$ and $\sigma^{0}: X^{0} \rightarrow X^{0}$ is the identity function, then we call $\sigma$ an edge-morphism, and, for notational convenience, identify $\sigma$ with $\sigma^{1}$.

A semigroupoid $S$ is a small graph $S$, together with a partial multiplication on the edges of $S$ such that, for any two edges $e, f \in S^{1}$

(i) the product $e f$ is defined if and only if $e \omega=f \alpha$;

(ii) if $e f$ is defined, then $(e f) \alpha=e \alpha$ and $(e f) \omega=f \omega$; and

(iii) if $e f$ and $f g$ are defined then $e(f g)=(e f) g$.

The vertices and edges of a semigroupoid are called respectively objects and arrows. Where no ambiguity arises, we abuse the notation slightly by writing $S$ to mean the set $S^{1}$ of arrows in $S$.

If $s_{0} \in S^{0}$ is such that there exists an $s \in S^{1}$ with $s \alpha=s \omega=s_{0}$, we say that the local semigroup of $S$ at the object $s_{0}$ is the semigroup with elements $\left\{s \in S \mid s \alpha=s \omega=s_{0}\right\}$ and multiplication defined by restricting that in $S$. A semigroupoid is strongly connected if for every pair $s_{0}, t_{0} \in S^{0}$ of objects, there is an arrow (or equivalently, a non-empty path) in $S$ with source $s_{0}$ and target $t_{0}$. An object $x \in S^{0}$ of a semigroupoid $S$ is isolated if is not the source or target of any arrow; if a semigroupoid has no isolated objects then it is isolation-free.

The free semigroupoid $X^{+}$on a small graph $X$ is the semigroupoid whose objects are the vertices of $X$, and whose arrows are the non-empty paths in $X$, with $\alpha, \omega$ and the partial multiplication defined in the obvious way. The free category $X^{*}$ is the category obtained by adjoining a local identity (which can be thought of as the empty path) at each object of $X^{+}$. A path language or just a language over $X$ is a (possibly empty) collection of (possibly empty) paths in $X$, that is, a subset of the free category $X^{*}$.

Let $x=x_{1} \ldots x_{n}$ be a path in a free semigroupoid $X^{+}$where each $x_{i} \in$ $X^{1}$. A prefix of $x$ is a (non-empty) path of the form $x_{1} \ldots x_{j}$ for some $1 \leq j \leq n$. A suffix of $x$ is a (non-empty) path of the form $x_{j} \ldots x_{n}$ for some $1 \leq j \leq n$. A factor of $x$ is a (non-empty) path of the form $x_{j} \ldots x_{k}$ for some $1 \leq j \leq k \leq n$. An internal factor of $x$ is a (non-empty) path of the form $x_{j} \ldots x_{k}$ for some $1<j \leq k<n$, that is, a factor of $x_{2} \ldots x_{n-1}$.

Given a path language $L \subseteq X^{+}$, we write $\operatorname{Pref}(L)$ [respectively $\operatorname{Suff}(L)$, Fact $(L), \operatorname{Int}(L)]$ for the set of non-empty prefixes [respectively suffixes, factors, internal factors] of paths in $L$. For $n \in \mathbb{N}$ we write $\operatorname{Pref}_{n}(L)$ to denote $\operatorname{Pref}(L) \cap X^{n}$, and similarly for $\operatorname{Suff}_{n}(L), \operatorname{Fact}_{n}(L)$ and $\operatorname{Int}_{n}(L)$; note that all four are empty when $n=0$.

A (path) automaton $M$ over a small graph $X$ is a small graph $M$, together with a graph morphism $\sigma: M \rightarrow X$, a set of distinguished start vertices of $M$ and a set of distinguished terminal vertices of $M$. We think of the automaton as the graph $M$ with each edge $e \in M^{1}$ labelled by $e \sigma^{1} \in X^{1}$, and each vertex $v \in M^{0}$ labelled by $v \sigma^{0} \in X^{0}$. The vertices and edges are called states and transitions respectively. The unique language accepted or language recognised by $M$ is the set of paths in $X$ which label paths from a start vertex to a terminal vertex in $M$.

The automaton is called a complete, deterministic automaton if (i) there is exactly one start vertex in the pre-image of each object in $X$ and (ii) for every state $m \in M^{0}$ and every edge $e \in X^{1}$ with $e \alpha=m \sigma^{0}$ there is a unique edge $f \in M^{1}$ with $f \sigma^{1}=e$ and $f \alpha=m$. The automaton is called finite if 
the graphs $M$ and $X$ are finite; we shall be concerned exclusively with finite automata.

A path language which is accepted by some finite path automaton is called regular. We recall from [17, Section 3] that a language $L \subseteq X^{+} \subseteq\left(X^{1}\right)^{+}$is regular in this sense if and only if it is regular in the usual sense as a language over the alphabet $X^{1}$. We recall also that the set of regular path languages over $X$ contains $X^{+}, X^{*}$ and all finite path languages, and is closed under concatenation, finite intersection, finite union, complement, set difference, generation of subcategories and subsemigroupoids, and prefix-closure. We will use these properties without further comment.

Given a graph $X$, we denote by $X^{\$}$ the graph $X$ with an extra edge $\$_{s}$ adjoined for every vertex $s \in X^{0}$, with source and target $s$. We define a function $\delta_{X}:\left(X^{+} \times X^{+}\right) \rightarrow\left(X^{\$} \times X^{\$}\right)^{+}$by

$$
(a, b) \delta_{X}= \begin{cases}\left(a_{1}, b_{1}\right) \ldots\left(a_{n}, b_{n}\right) & \text { if } m=n \\ \left(a_{1}, b_{1}\right) \ldots\left(a_{n}, b_{n}\right)\left(a_{n+1}, \$_{b_{n} \omega}\right) \ldots\left(a_{m}, \$_{b_{n} \omega}\right) & \text { if } m>n \\ \left(a_{1}, b_{1}\right) \ldots\left(a_{m}, b_{m}\right)\left(\$_{a_{m} \omega}, b_{n+1}\right) \ldots\left(\$_{a_{m} \omega}, b_{n}\right) & \text { if } n>m\end{cases}
$$

where $a=a_{1} \ldots a_{m}$ and $b=b_{1} \ldots b_{n}$. We observe that the function $\delta_{X}$ is injective, and in particular that it distributes over intersection, that is, that

$$
\left(R_{1} \cap R_{2}\right) \delta_{X}=R_{1} \delta_{X} \cap R_{2} \delta_{X}
$$

for all binary relations $R_{1}, R_{2} \subseteq X^{+} \times X^{+}$.

A (binary) synchronous path automaton over $X$ is a finite path automaton over the graph $X^{\$} \times X^{\$}$, with the property that the language accepted is contained within the image $\left(X^{+} \times X^{+}\right) \delta_{X}$ of $\delta_{X}$. For convenience, we shall say that a synchronous path automaton accepts a pair $(a, b) \in X^{+} \times X^{+}$if it accepts $(a, b) \delta_{X}$. A binary relation $R \subseteq X^{+} \times X^{+}$is called synchronously regular if there exists a synchronous path automaton accepting exactly the language $R \delta_{X}$, that is, if $R \delta_{X}$ is regular.

Lemma 2.1. [17, Lemma 3.7] Let $X$ be a finite graph. Then:

(i) If $R \subseteq X^{+} \times X^{+}$is synchronously regular, then the binary relation

$$
R^{-1}=\{(v, u) \mid(u, v) \in R\}
$$

is synchronously regular.

(ii) If $R \subseteq X^{+} \times X^{+}$is synchronously regular, then the projections $R \pi_{1}$ and $R \pi_{2}$ of $R$ onto its first and second coordinates are regular.

(iii) If $K, L \subseteq X^{+}$are regular languages of non-empty paths, then the binary relation $K \times L$ is synchronously regular.

(iv) Synchronously regular binary relations over $X$ are closed under finite union, finite intersection and relational composition.

(v) If $K \subseteq X^{+}$is regular then the diagonal binary relation

$$
\{(w, w) \mid w \in K\}
$$

is synchronously regular.

Let $X$ and $Y$ be finite graphs, $A \subseteq X^{*}$ and $\phi: A \rightarrow Y^{*}$ be a function. We say that $\phi$ is strongly regularity preserving if for every regular language $L \subseteq X^{+}$, we have that $(L \cap A) \phi \subseteq Y^{+}$is a regular language. 
Let $X$ and $Y$ be finite graphs, and for $i=1,2$ suppose we have subsets $A_{i} \subseteq X^{+}$and functions $\phi_{i}: A_{i} \rightarrow Y^{+}$. We say that $\phi_{1}$ and $\phi_{2}$ are strongly mutually synchronous regularity preserving if for every synchronously regular relation $R \subseteq X^{+} \times X^{+}$, the relation

$$
\left\{\left(u \phi_{1}, v \phi_{2}\right) \mid(u, v) \in R \cap\left(A_{1} \times A_{2}\right)\right\} \subseteq Y^{+} \times Y^{+}
$$

is synchronously regular.

A sliding window inverse for a function $\phi: A \rightarrow Y^{+}$is a quadruple $(n, f, g, h)$ consisting of a positive integer $n$ and three functions

$$
\begin{gathered}
f: \operatorname{Pref}_{n}\left(A \phi \cap Y^{>n}\right) \rightarrow X^{*}, \\
g: \operatorname{Int}_{n}(A \phi) \rightarrow X^{*} \text { and } \\
h: \operatorname{Suff}_{n}\left(A \phi \cap Y^{>n}\right) \rightarrow X^{*},
\end{gathered}
$$

with the property that for any $w \in A$ and $y_{1} \ldots y_{k} \in Y^{+}$with $y_{1}, \ldots, y_{k} \in Y^{1}$ and $k>n$ such that $w \phi=y_{1} \ldots y_{k}$, we have

$$
\begin{aligned}
w=\left(y_{1} \ldots y_{n}\right) f\left(y_{2} \ldots y_{n+1}\right) g\left(y_{3} \ldots y_{n+2}\right) g \ldots \\
\ldots\left(y_{k-n} \ldots y_{k-1}\right) g\left(y_{k-n+1} \ldots y_{k}\right) h .
\end{aligned}
$$

If $f, g$ and $h$ are functions with domains containing those given above, we shall say that $(n, f, g, h)$ is a sliding window inverse for $\phi$ if the restrictions of $f, g$ and $h$ to the appropriate domains have the given properties.

Lemma 2.2. 17, Lemma 3.8] Let $X$ and $Y$ be finite graphs, $A \subseteq X^{*}$ and $\phi: A \rightarrow Y^{*}$ be a function. If $A \phi \subseteq Y^{*}$ is regular, and $\phi$ has a sliding window inverse, then $\phi$ is strongly regularity preserving.

Let $\psi: \mathbb{N} \rightarrow \mathbb{N}$ be a function. We say that sliding window inverses $\left(n_{1}, f_{1}, g_{1}, h_{1}\right)$ for $\phi_{1}$ and $\left(n_{2}, f_{2}, g_{2}, h_{2}\right)$ for $\phi_{2}$ are synchronised by $\psi$ if

(i) $n_{1}=n_{2}$;

(ii) for every $i=1,2$ and every $w \in \operatorname{Pref}_{n_{1}}\left(A_{i} \phi_{i} \cap Y^{>n_{1}}\right)$, we have $\left|w f_{i}\right|=0 \psi$

(iii) for every $i=1,2$ and every $x y z \in A_{i} \phi_{i}$ with $x \in Y^{m}, m \geq 1, y \in Y^{n_{1}}$ and $z \in Y^{+}$we have $\left|y g_{i}\right|=m \psi$; and

(iv) for every $i=1,2$ and every $x y \in A_{i} \phi_{i}$ with $x \in Y^{m}, m \geq 1$ and $y \in Y^{n_{1}}$ we have $\left|y h_{i}\right| \leq m \psi$.

We say that two sliding window inverses are synchronised if they are synchronised by some function $\psi: \mathbb{N} \rightarrow \mathbb{N}$.

Lemma 2.3. 17, Lemma 3.9] Let $X$ and $Y$ be finite graphs, $A_{1}, A_{2} \subseteq X^{+}$ and $\phi_{1}: A_{1} \rightarrow Y^{+}$and $\phi_{2}: A_{2} \rightarrow Y^{+}$be injective functions. If $A_{1} \phi_{1}$ and $A_{2} \phi_{2}$ are regular and $\phi_{1}$ and $\phi_{2}$ have synchronised sliding window inverses, then $\phi_{1}$ and $\phi_{2}$ are strongly mutually synchronous regularity preserving.

A choice of representatives for a semigroupoid $S$ is a triple $(X, K, \rho)$, of a graph $X$ with $X^{0}=S^{0}$, a (surjective) semigroupoid morphism $\rho: X^{+} \rightarrow S$ of the free semigroupoid $X^{+}$onto $S$, and a language $K \subseteq X^{+}$such that $K \rho=S$. The choice of representatives is called finitely generated if $X$ has finitely many edges. Clearly, a semigroupoid has a finitely generated choice of representatives if and only if it is finitely generated. The choice of representatives is called a cross-section if the restriction of $\rho$ to $K$ is 
bijective, that is, if $K$ contains a unique representative for every arrow in $S$. The choice of representatives is called prefix-closed if $K$ is closed under the taking of non-empty prefixes.

An automatic structure for a semigroupoid $S$ is a finitely generated choice of representatives $(X, K, \rho)$ with the property that for every edge or empty path $a \in X^{0} \cup X^{1}$, the binary relation

$$
K_{a}=\{(u, v) \in K \times K \mid u \omega=a \alpha,(u a) \rho=v \rho\}
$$

is synchronously regular. Equivalently [17, Proposition 4.1], $(X, K, \rho)$ is an automatic structure exactly if $K_{a}$ is synchronously regular for every $a \in X^{1}$, and the union

$$
K_{=}=\{(u, v) \in K \times K \mid u \rho=v \rho\}=\bigcup_{a \in X^{0}} K_{a}
$$

is synchronously regular.

A prefix-automatic structure for a semigroupoid $S$ is an automatic structure $(X, K, \rho)$ with the additional property that the binary relation

$$
K_{=}^{\prime}=\{(u, v) \in K \times \operatorname{Pref}(K) \mid u \rho=v \rho\}
$$

is synchronously regular. A semigroupoid admits a prefix-automatic structure if and only if it admits an automatic structure which is prefix-closed 17. Corollary 4.6].

Now let $S$ be a semigroupoid and 0 be a new symbol not in $S^{1}$. The consolidation of $S$ is the semigroup with set of elements $T=S^{1} \cup\{0\}$, and multiplication defined by

$$
s t= \begin{cases}\text { the } S \text {-product } s t & \text { if } s, t \in S \text { and } s \omega=t \alpha \\ 0 & \text { otherwise }\end{cases}
$$

for all $s, t \in S$. We shall need the following key results from [17.

Theorem 2.4. 17, Theorem 4.3] Let $T$ be the consolidation of a semigroupoid $S$. Then $S$ is automatic [prefix-automatic] if and only if $T$ is automatic [respectively, prefix-automatic].

Theorem 2.5. 17, Theorem 5.6] Let $S$ be a semigroupoid and $U$ a cofinite subsemigroupoid of $S$. Then $S$ is automatic [prefix-automatic] if and only if $U$ is automatic [respectively, prefix-automatic].

\section{Some Technical Results}

We shall need the following technical results in the remaining sections.

Proposition 3.1. Let $X$ be a finite graph and $R \subseteq X^{+} \times X^{+}$be synchronously regular. Let $m \geq 0$ and let $f: X^{m} \rightarrow X^{*}$ be such that $x f \alpha=x \alpha$ for all $x \in X^{m}$. Then the binary relation

$$
R^{\prime}=\left\{(u, v(x f)) \mid(u, v x) \in R, u, v \in X^{*}, x \in X^{m}\right\}
$$

is synchronously regular.

Proof. First, notice that $R^{\prime}$ is the composition of $R$ with the relation

$$
T=\left\{(v x, v(x f)) \mid v \in X^{*}\right\} .
$$


By Lemma 2.1, it will suffice to show that $T$ is synchronously rational. But clearly $T \delta_{X}$ is the concatenation of $D \delta_{X}$ with $E \delta_{X}$ where $D$ is the diagonal relation on $X$ and $E$ is the finite relation $\left\{(x, x f) \mid x \in X^{m}\right\}$. Both of these are regular, and regular path languages are closed under concatenation, so it follows that $T \delta_{X}$ is regular, and so $T$ is synchronously regular as required.

Proposition 3.2. Let $S$ be a semigroupoid with an automatic cross-section [prefix-closed automatic structure, prefix-automatic cross-section], and let $T$ be a finite subset of $S$. Then $S$ has an automatic cross-section [prefix-closed automatic structure, prefix-automatic cross-section] $(Y, L, \sigma)$ such that the restriction of $\sigma$ to $Y$ is injective and has image containing $T$.

Proof. Let $(X, K, \rho)$ be an automatic cross-section [prefix-closed automatic structure, prefix-automatic cross-section] for $S$. Choose a subgraph $Y$ of $X$ such that $Y^{1} \rho=X^{1} \rho$ and the restriction of $\rho$ to $Y^{1}$ is injective. For each element $t \in T$ which does not already have a representative in $X$, adjoin a new edge $y_{t}$ to $Y$ with $y_{t} \alpha=t \alpha$ and $y_{t} \omega=t \omega$, to obtain a new finite graph $Z$.

For each $x \in X^{1}$, let $x \sigma$ be the unique element $y \in Z$ such that $y \rho=x \rho$. Extend $\sigma$ to a semigroupoid morphism $\sigma^{\prime}: X^{+} \rightarrow Z^{+}$. Let $\rho^{\prime}: Z^{+} \rightarrow S$ be defined by

$$
x \rho^{\prime}= \begin{cases}x \rho & \text { if } x \in X \\ t & \text { if } x=y_{t} .\end{cases}
$$

It is a routine exercise to verify that $\left(Z, K \sigma^{\prime}, \rho^{\prime}\right)$ is an automatic cross-section [prefix-closed automatic structure, prefix-automatic cross-section] for $S$ and that the restriction of $\rho^{\prime}$ to $Z$ is injective and has image containing $T$.

Lemma 3.3. Let $(X, K, \sigma)$ be a regular choice of representatives for a semigroupoid $S$, and let $L$ be a cofinite subset of $K$. If $K_{=} \cap(L \times L)$ is synchronously regular and $K_{c} \cap(L \times K)$ is synchronously regular for every $c \in X^{1}$, then $(X, K, \sigma)$ is an automatic structure for $S$.

Proof. First, we claim that for any $w \in K$, the language

$$
w \sigma \sigma^{-1} \cap K=\{x \in K \mid x \sigma=w \sigma\}
$$

is regular. If $L$ contains no representatives for $w \sigma$, then $w \sigma \sigma^{-1} \cap K \subseteq K \backslash L$ must be finite and hence regular. Otherwise, let $u$ be a representative in $L$ for $w \sigma$. Now we have

$$
w \sigma \sigma^{-1} \cap K=\left(w \sigma \sigma^{-1} \cap(K \backslash L)\right) \cup\left(\left(K_{=} \cap(L \times L)\right) \cap(\{u\} \times L)\right) \pi_{2}
$$

where $w \sigma \sigma^{-1} \cap K \backslash L \subseteq K \backslash L$ is finite, $K_{=} \cap(L \times L)$ is synchronously regular by assumption, and $L$ is regular. It follows by Lemma 2.1 that $w \sigma \sigma^{-1} \cap K$ is regular. Now we see that

$$
\begin{aligned}
K_{=}=\left(K_{=} \cap(L \times L)\right) \cup & \left(\bigcup_{w \in K \backslash L}\{w\} \times\left(w \sigma \sigma^{-1} \cap K\right)\right) \cup \\
& \left(\bigcup_{w \in K \backslash L}\left(w \sigma \sigma^{-1} \cap K\right) \times\{w\}\right)
\end{aligned}
$$


is synchronously regular by Lemma 2.1(iii) and (iv). Similarly, for any $c \in C^{1}$ we see that

$$
K_{c}=\left(K_{c} \cap(L \times K)\right) \cup \bigcup_{w \in K \backslash L}\{w\} \times\left((w c) \sigma \sigma^{-1} \cap K\right)
$$

is synchronously regular by Lemma 2.1(iii) and (iv). Thus, $(X, K, \sigma)$ is an automatic structure for $S$, as required.

Lemma 3.4. Let $(X, L, \sigma)$ be an automatic structure for a semigroupoid $S$, and suppose $L \subseteq K \subseteq X^{+}$with $K \backslash L$ finite. Then $(X, K, \sigma)$ is an automatic structure for $S$.

Proof. First, note that $K_{=} \cap(L \times L)=L_{=}$is synchronously regular and $K_{c} \cap(L \times L)=L_{c}$ is synchronously regular for every $c \in X^{1}$. We can deduce as in the proof of Lemma 3.3 that $w \sigma \sigma^{-1} \cap K$ is regular for every $w \in K$, and that $K_{=}$is synchronously regular.

Now let $c \in X^{1}$. For any $w \in K$, choose $u \in L$ such that $w \sigma=u \sigma$, and consider the set

$$
\begin{aligned}
& \{x \in K \mid x \omega=c \alpha,(x c) \sigma=w \sigma\} \\
= & \{x \in K \backslash L \mid x \omega=c \alpha,(x c) \sigma=w \sigma\} \cup\{x \in L \mid x \omega=c \alpha,(x c) \sigma=w \sigma\} \\
= & \{x \in K \backslash L \mid x \omega=c \alpha,(x c) \sigma=w \sigma\} \cup\left(\left(K_{c} \cap(L \times L)\right) \cap(L \times\{u\})\right) \pi_{1} .
\end{aligned}
$$

Certainly the set $\{x \in K \backslash L \mid x \omega=c \alpha,(x c) \sigma=w \sigma\}$ is finite, and we know that $K_{c} \cap(L \times L)$ is synchronously regular and $L$ is regular. It follows by Lemma 2.1(ii) (iii) and (iv) that $\{x \in K \mid x \omega=c \alpha,(x c) \sigma=w \sigma\}$ is regular.

It follows that

$$
\begin{aligned}
K_{c}=\left(K_{c} \cap(L \times L)\right) \cup & \bigcup_{w \in K \backslash L}\{w\} \times\left((w c) \sigma \sigma^{-1} \cap K\right) \cup \\
& \bigcup_{w \in K \backslash L}\{x \in K \mid x \omega=c \alpha,(x c) \sigma=w \sigma\} \times\{w\}
\end{aligned}
$$

is synchronously regular by Lemma 2.1(iii) and (iv). Thus, $(X, K, \sigma)$ is an automatic structure for $S$, as required.

\section{Rees Matrices and Automaticity}

Rees matrix constructions over semigroupoids were introduced by Lawson [19], who used them to construct a class of locally adequate abundant semigroups. They represent an alternative formulation of certain special cases of the blocked Rees matrix semigroup constructions introduced by Fountain [8] and subsequently employed by Armstrong [1]. In this section, we extend some results of [5] concerning Rees matrix constructions over semigroups to cover similar cosntructions over semigroupoids and small categories. In some cases, our new results are stronger than previously known results, even when specialised to the case of semigroups.

Let $S$ be a non-empty, isolation-free semigroupoid, and $I$ and $\Lambda$ be indexing sets. Let $F: I \rightarrow S^{1} \alpha \subseteq S^{0}$ and $G: \Lambda \rightarrow S^{1} \omega \subseteq S^{0}$ be surjective functions. Let 0 be a new symbol not in $S$, and let $P$ be a $\Lambda \times I$ matrix with entries drawn from $S \cup\{0\}$, with the property that $P_{\lambda i} \omega=i F$ and 
$P_{\lambda i} \alpha=\lambda G$ for all $i \in I, \lambda \in \Lambda$ such that $P_{\lambda i} \neq 0$. The Rees matrix semigroup with zero $M=M^{0}(S ; F(I), G(\Lambda) ; P)$ is the semigroup with set of elements

$$
M=\{0\} \cup\{(i, x, \lambda) \in I \times S \times \Lambda \mid i F=x \alpha, x \omega=\lambda G\}
$$

and multiplication given by

$$
(i, x, \lambda)(j, y, \mu)= \begin{cases}\left(i, x P_{\lambda j} y, \mu\right) & \text { if } P_{\lambda j} \neq 0 \\ 0 & \text { otherwise }\end{cases}
$$

for all $(i, x, \lambda),(j, y, \mu) \in M \backslash\{0\}$, and $0 m=m 0=0$ for all $m \in M$.

Note that in the expression " $M^{0}(S ; F(I), G(\Lambda) ; P)$ ", the use of the notation " $F(I)$ " and " $G(\Lambda)$ " is purely symbolic. It is intended to remind the reader of the relationship between $F$ and $I$ and between $G$ and $\Lambda$.

We call $P$ the sandwich matrix of the construction. If $P$ contains no zero entries, then $M \backslash\{0\}$ is a subsemigroup of $M$, which we call the Rees matrix semigroup (without zero) $M(S ; F(I), G(\Lambda) ; P)$.

We remark briefly upon the choice of codomains for the indexing functions $F$ and $G$. This restriction does not limit the range of semigroups which appear as Rees matrix semigroups. Indeed, if $I, \Lambda, F, G, S$ and $P$, do not satisfy this requirement but otherwise satisfy the requirements for the Rees matrix construction, then one can instead perform the construction with subsets of $I$ and $\Lambda$, a subsemigroupoid of $S$ and the corresponding restrictions of $F$ and $G$ and submatrix of $P$, to obtain the same Rees matrix semigroup as would be obtained by using the construction on the more general semigroupoid. Similarly, given a semigroupoid $S$ with isolated objects, one can remove those objects to obtain an isolation-free semigroupoid before using a Rees matrix construction. The purpose of the restrictions is to allow the following straightforward proposition.

Proposition 4.1. Let

$$
M=M^{0}(S ; F(I), G(\Lambda) ; P)
$$

or

$$
M=M(S ; F(I), G(\Lambda) ; P)
$$

be a Rees matrix semigroup with or without zero over a semigroupoid $S$. Then

- for every $i \in I$ there exist $s \in S^{1}$ and $\lambda \in \Lambda$ with $(i, s, \lambda) \in M$;

- for every $s \in S^{1}$ there exist $i \in I$ and $\lambda \in \Lambda$ with $(i, s, \lambda) \in M$;

- for every $\lambda \in \Lambda$ there exist $i \in I$ and $s \in S^{1}$ with $(i, s, \lambda) \in M$; and

- for every $v \in S^{0}$ there exists $i \in I$ with $i F=v$ or $\lambda \in \Lambda$ with $\lambda G=v$.

The following theorem is an amalgamation of results which can be found in [16]; the same results appear, in slightly less generality, in [18]. For a precise definition of what it means for a semigroupoid to be finitely presentable, the reader is directed to [18.

Theorem 4.2. Let

$$
M=M(S ; F(I), G(\Lambda) ; P)
$$


or

$$
M=M^{0}(S ; F(I), G(\Lambda) ; P)
$$

be a Rees matrix semigroup (with or without zero) over an isolation-free semigroupoid $S$. Then $M$ is finitely generated [finitely presentable] if and only if

(i) the indexing sets $I$ and $\Lambda$ are finite;

(ii) the semigroupoid $S$ is finitely generated [respectively, finitely presentable]; and

(iii) $S P^{\prime} S$ is a cofinite subsemigroupoid of $S$, where $P^{\prime}$ is the set of nonzero entries in the sandwich matrix $P$.

In order for a semigroup or semigroupoid to be automatic it is, of course, necessary that it be finitely generated. Theorem 4.2 gives necessary and sufficient conditions for a Rees matrix semigroup over a finitely generated semigroupoid to be finitely generated. We begin by showing that the same conditions suffice to ensure that a Rees matrix semigroup over an automatic small category is automatic. We shall then extend this result to cover Rees matrix semigroups over general automatic semigroupoids.

Theorem 4.3. Let $M=M^{0}(S ; F(I), G(\Lambda) ; P)$ be a Rees matrix semigroup with zero over a small category $S$. Suppose $S$ is automatic (as a semigroupoid) and $M$ is finitely generated. Then $M$ is automatic.

Proof. Let $P^{\prime}$ be the set of non-zero entries in the sandwich matrix $P$, and let $U=S P^{\prime} S$. Since $M$ is finitely generated and $S$ is a small category, we deduce, by Theorem 4.2 that $I$ and $\Lambda$ are finite and that $U$ is a cofinite subsemigroupoid of $S$. It follows by Theorem 2.5 that $U$ is automatic, by [17. Corollary 4.6] that $U$ has an automatic cross-section, and then by Proposition 3.2 that $U$ has an automatic cross-section $(X, K, \sigma)$ with the property that the restriction of $\sigma$ to $X$ is injective.

By the definition of $U$, for each $y \in X$ we can choose $s_{y}, t_{y} \in S, i_{y} \in I$ and $\lambda_{y} \in \Lambda$ such that $y \sigma=s_{y} P_{\lambda_{y} i_{y}} t_{y}$. Now let

$$
H=\left\{t_{y}, s_{y} \mid y \in X\right\} \cup\left\{1_{s} \mid s \in S^{0}\right\} \subseteq S .
$$

We define new alphabets

$$
A=\left\{a_{i g h \lambda} \mid i \in I, g, h \in H, \lambda \in \Lambda, i F=g \alpha, g \omega=h \alpha, h \omega=\lambda G\right\}
$$

and

$$
B=\left\{b_{i s \lambda} \mid i \in I, s \in S \backslash U, \lambda \in \Lambda, i F=s \alpha, s \omega=\lambda G\right\} .
$$

Clearly, $A$ and $B$ are finite. Let $z$ be a new symbol not in $A$ or $B$, which will represent the zero element $0 \in M$. We define a morphism of semigroups

$$
\rho:(A \cup B \cup\{z\})^{+} \rightarrow M
$$

by $\left(a_{i g h \lambda}\right) \rho=(i, g h, \lambda),\left(b_{i s \lambda}\right) \rho=(i, s, \lambda)$ and $z \rho=0$.

For every $i \in I$ and $\lambda \in \Lambda$, let $X_{i \lambda}$ be the set

$$
X_{i \lambda}=\left\{x \in X^{+} \mid i F=x \alpha, x \omega=\lambda G\right\}
$$

of words in $X^{+}$starting at $i F$ and finishing at $\lambda G$, and define a function $\phi_{i \lambda}: X_{i \lambda} \rightarrow A^{+}$by

$$
\left(w_{1} w_{2} \ldots w_{n}\right) \phi_{i \lambda}=a_{i, 1_{i F}, s_{w_{1}}, \lambda_{w_{1}}} a_{i_{w_{1}}, t_{w_{1}}, s_{w_{2}}, \lambda_{w_{2}}} \ldots a_{i_{w_{n}}, t_{w_{n}}, 1_{\lambda G}, \lambda}
$$


for all $w_{1}, w_{2}, \ldots, w_{n} \in X$ with $w_{1} \ldots w_{n} \in X_{i \lambda}$. It follows from the fact that the restriction of $\sigma$ to $X$ is injective, that each function $\phi_{i \lambda}$ is injective. Indeed, if $\left(w_{1} \ldots w_{n}\right) \phi_{i \lambda}=\left(w_{1}^{\prime} \ldots w_{n^{\prime}}^{\prime}\right) \phi_{i \lambda}$ then from the definition of $\phi_{i \lambda}$ we have

$$
\begin{aligned}
& a_{i, 1_{i F}, s_{w_{1}}, \lambda_{w_{1}}} a_{i_{w_{1}}, t_{w_{1}}, s_{w_{2}}, \lambda_{w_{2}}} \ldots a_{i_{w_{n}}, t_{w_{n}}, 1_{\lambda G}, \lambda}=\left(w_{1} \ldots w_{n}\right) \phi_{i \lambda} \\
= & \left(w_{1}^{\prime} \ldots w_{n^{\prime}}^{\prime}\right) \phi_{i \lambda}=a_{i, 1_{i F}, s_{w_{1}^{\prime}}, \lambda_{w_{1}^{\prime}}} a_{i_{w_{1}^{\prime}}, t_{w_{1}^{\prime}}, s_{w_{2}^{\prime}}, \lambda_{w_{2}^{\prime}}} \ldots a_{i_{w_{n^{\prime}}^{\prime}}, t_{w_{n^{\prime}}^{\prime}}, 1_{\lambda G}, \lambda}
\end{aligned}
$$

so clearly $n=n^{\prime}$ and for $1 \leq k \leq n$ we have $s_{w_{k}}=s_{w_{k}^{\prime}}, i_{w_{k}}=i_{w_{k}^{\prime}}, \lambda_{w_{k}}=\lambda_{w_{k}^{\prime}}$ and $t_{w_{k}}=t_{w_{k}^{\prime}}$. Now each

$$
w_{k} \sigma=s_{w_{k}} P_{\lambda_{w_{k}} i_{w_{k}}} t_{w_{k}}=s_{w_{k}^{\prime}} P_{\lambda_{w_{k}^{\prime}}{ }^{i} w_{k}^{\prime}} t_{w_{k}^{\prime}}=w_{k}^{\prime} \sigma .
$$

But $\sigma$ is by assumption injective on $X$, so it follows that each $w_{k}=w_{k}^{\prime}$.

Note also that

$$
(i, w \sigma, \lambda)=w \phi_{i \lambda} \rho
$$

for all paths $w \in X_{i \lambda}$.

Our aim is to show that each function of the form $\phi_{i \lambda}$ is strongly regularity preserving, and that each pair of such functions is strongly mutually synchronous regularity preserving. We shall do so by showing that each such function has a regular image and that the functions have sliding window inverses which are pairwise synchronised, and then invoking Lemmas 2.2 and 2.3 .

We claim first that the image $X_{i \lambda} \phi_{i \lambda}$ of each $\phi_{i \lambda}$ is regular. We say that a two-letter word $a_{i_{1}, g_{1}, h_{1}, \lambda_{1}} a_{i_{2}, g_{2}, h_{2}, \lambda_{2}} \in A^{2}$ is compatible if there exists $y \in X$ such that $s_{y}=h_{1}, \lambda_{y}=\lambda_{1}, i_{y}=i_{2}$ and $t_{y}=g_{2}$, and incompatible otherwise. Then clearly, $X_{i \lambda} \phi_{i \lambda}$ is the set of words in $A^{\geq 2}$ which begin with a letter of the form $a_{i, 1_{i F}, h, \mu}$ for some $h \in H$ and $\mu \in \lambda$, end with a letter of the form $a_{j, g, 1_{\lambda G}, \lambda}$ for some $j \in I$ and $g \in H$, and contain no incompatible factors of length 2. Thus, $X_{i \lambda} \phi_{i \lambda}=\left(A_{1} A^{*} A_{2}\right) \backslash\left(A^{*} A_{3} A^{*}\right)$ where

$$
\begin{gathered}
A_{1}=\left\{a_{i, 1_{i F}, h, \mu} \in A \mid h \in H, \mu \in \Lambda, i F=h \alpha, h \omega=\mu G\right\}, \\
A_{2}=\left\{a_{j, g, 1_{\lambda G}, \lambda} \in A \mid j \in I, g \in H, j F=g \alpha, g \omega=\lambda G\right\}, \text { and } \\
A_{3}=\left\{a b \in A^{2} \mid a b \text { is not compatible }\right\}
\end{gathered}
$$

are all finite. It follows that $X_{i \lambda} \phi_{i \lambda}$ is regular, as required.

Next, we claim that the $\phi_{i \lambda}$ have pairwise synchronised sliding window inverses. Fix $i \in I$ and $\lambda \in \Lambda$. We shall define a function

$$
f:\left\{a b \in A^{2} \mid a b \text { is compatible }\right\} \rightarrow X .
$$

By our observations above, the domain of $f$ will then include all two-letter factors of words in $X_{i \lambda} \phi_{i \lambda}$.

Suppose $a_{i_{1} g_{1} h_{1} \lambda_{1}} a_{i_{2} g_{2} h_{2} \lambda_{2}}$ is compatible. Then by definition, there is some $y \in X$ with $s_{y}=h_{1}, \lambda_{y}=\lambda_{1}, i_{y}=i_{2}$ and $t_{y}=g_{2}$. Furthermore, we have $y \sigma=h_{1} P_{\lambda_{1} i_{2}} g_{2}$, and $\sigma$ is injective on $X$, so $y$ is the unique letter in $X$ with this property. Thus, we can well-define $f$ by the rule

$$
\left(a_{i_{1} g_{1} h_{1} \lambda_{1}} a_{i_{2} g_{2} h_{2} \lambda}\right) f=y .
$$


We claim that $(2, f, f, f)$ is a sliding window inverse for $\phi_{i \lambda}$. To show this, suppose $w=w_{1} w_{2} \ldots w_{n} \in X_{i \lambda}$ with $w_{1}, \ldots, w_{n} \in X$. Then by the definition of $\phi_{i \lambda}$ we have

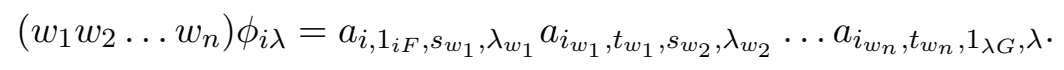

Let

$$
y_{1}=a_{i, 1_{i F}, s_{w_{1}}, \lambda_{w_{1}}}, \quad y_{2}=a_{i_{w_{1}}, t_{w_{1}}, s_{w_{2}}, \lambda_{w_{2}}}, \quad \ldots, \quad y_{n+1}=a_{i_{w_{n}}, t_{w_{n}}, 1_{\lambda G}, \lambda}
$$

so that

$$
\left(w_{1} w_{2} \ldots w_{n}\right) \phi_{i \lambda}=y_{1} y_{2} \ldots y_{n+1} \text {. }
$$

Now from the definition of $f$, we see that $\left(y_{i} y_{i+1}\right) f=w_{i}$ for each $1 \leq i \leq n$, so we have

$$
\left(y_{1} y_{2}\right) f\left(y_{2} y_{3}\right) f\left(y_{3} y_{4}\right) f \ldots\left(y_{n-1} y_{n}\right) f\left(y_{n} y_{n+1}\right) f=w_{1} \ldots w_{n}
$$

as required. It follows by Lemma 2.2 that each $\phi_{i \lambda}$ is strongly regularity preserving.

Furthermore, if we define $\psi: \mathbb{N} \rightarrow \mathbb{N}$ to be the constant function given by $n \psi=1$, then $m \psi=1=|w f|$ for any $w \in \operatorname{Fact}_{2}\left(X_{i \lambda} \phi_{i \lambda}\right)$ which occurs starting in position $m$ (numbered from zero). Since the definition of $\psi$ is independent of the choice of $i$ and $\lambda$, it follows that $\psi$ synchronises the sliding window inverses constructed above for any pair of functions of the form $\phi_{i \lambda}$. We have already observed that the $\phi_{i \lambda}$ are injective and have regular images, so it follows by Lemma 2.3 that any pair of such functions are strongly mutually synchronous regularity preserving.

We now define a language $L \subseteq(A \cup B \cup\{z\})^{+}$by

$$
L=B \cup\{z\} \cup \bigcup_{i \in I, \lambda \in \Lambda}\left(K \cap X_{i \lambda}\right) \phi_{i \lambda}
$$

We claim that $(A \cup B \cup\{z\}, L, \rho)$ is an automatic structure (indeed, an automatic cross-section) for $M$.

We show first that $L$ contains a unique representative for every element of $M$. Certainly $z \in L$ is the unique word representing the zero element $0 \in M$. Now consider a non-zero element $(i, s, \lambda) \in M$. If $s \in S \backslash U$ then $b_{i s \lambda} \in B$ is clearly the unique word in $L$ representing $(i, s, \lambda)$, as required. Otherwise, we have $s \in U$. Now $(X, K, \sigma)$ is a choice of representatives for $U$, so we must have $s=w \sigma$ for some path $w \in K$. Furthermore, $w \alpha=s \alpha=i F$ and $w \omega=s \omega=\lambda G$, so that $w \in X_{i \lambda}$. Now by (11) we have

$$
(i, s, \lambda)=(i, w \sigma, \lambda)=w \phi_{i \lambda} \rho
$$

where $w \phi_{i \lambda} \in\left(K \cap X_{i \lambda}\right) \phi_{i \lambda} \subseteq L$. Furthermore, if $i^{\prime} \in I$ and $\lambda^{\prime} \in \Lambda$ and $v \in K \cap X_{i^{\prime} \lambda^{\prime}}$ are such that $v \phi_{i^{\prime} \lambda^{\prime}}=(i, s, \lambda)$, then by (10) we must have $\left(i^{\prime}, v \sigma, j^{\prime}\right)=(i, w \sigma, j)$ from which it follows that $i=i^{\prime}, j=j^{\prime}$ and, since $K$ contains a unique representative for every element in $U, v=w$. Hence $w \phi_{i \lambda}$ is the unique representative for $(i, s, \lambda)$ in $L$. Thus, $(A \cup B \cup\{z\}, L, \rho)$ is a cross-section for $M$.

Next, we show that $L$ is a regular language. Clearly, $B$ and $\{z\}$ are finite and hence regular. Furthermore, $K$ is regular and each $\phi_{i \lambda}$ is strongly regularity preserving, so each $\left(K \cap X_{i \lambda}\right) \phi_{i \lambda}$ is regular. It follows that $L$ is a finite union of regular languages, and hence is regular. 
Since $(A \cup B \cup\{z\}, L, \rho)$ is a cross-section for $M$, it follows by Lemma2.1(v) that the binary relation

$$
L=\{(u, v) \in L \times L \mid u \rho=v \rho\}=\{(u, u) \mid u \in L\}
$$

is synchronously regular.

It remains to show that $L_{a}$ is synchronously regular for all $a \in A \cup B \cup\{z\}$. First, since $z$ is the unique representative in $L$ for the zero element $0 \in M$, we have $L_{z}=\{(w, z) \mid w \in L\}=L \times\{z\}$ which is synchronously regular by Lemma 2.1(iii).

Now suppose $a \in A \cup B$, with $a \rho=\left(i_{a}, s_{a}, \lambda_{a}\right)$. By Lemma 3.3. it will suffice to show that

$$
L_{a} \cap\left(\left(A^{+} \cap L\right) \times L\right)=L_{a} \cap\left(A^{+} \times L\right)
$$

is synchronously regular. We write $L_{a} \cap\left(A^{+} \times L\right)$ as a union

$$
\begin{aligned}
L_{a} \cap\left(A^{+} \times L\right)= & \left(L_{a} \cap\left(A^{+} \times B\right)\right) \\
& \cup\left(L_{a} \cap\left(A^{+} \times\{z\}\right)\right) \\
& \cup\left(L_{a} \cap\left(A^{+} \times A^{+}\right)\right),
\end{aligned}
$$

and show that each component in this union is synchronously regular.

Suppose $(u, v) \in L_{a} \cap\left(A^{+} \times L\right)$, so that $(u \rho)(a \rho)=v \rho$. Clearly $v \notin B$, since letters in $B$ represent indecomposable elements of $M$, so $L_{a} \cap\left(A^{+} \times B\right)$ is empty and hence synchronously regular.

If $v=z$, then we have $(u \rho)(a \rho)=v \rho=z \rho=0$. Since $u \in A^{+} \cap L$ cannot represent the zero element, this can happen only if $P_{u \rho \pi_{3}, a \rho \pi_{1}}=0$. Thus,

$$
\begin{aligned}
L_{a} \cap\left(A^{+} \times\{z\}\right) & =\left\{(u, z) \mid u \in A^{+}, P_{u \rho \pi_{3}, a \rho \pi_{1}}=0\right\} \\
& =\left(\bigcup_{b \in A, P_{\left(b \rho \pi_{3}\right), a \rho \pi_{1}}=0}\left(A^{*} b \cap L\right)\right) \times\{z\}
\end{aligned}
$$

is a product of finite unions of regular languages, and by Lemma 2.1(iii) is synchronously regular.

It remains only to show that $L_{a} \cap\left(A^{+} \times A^{+}\right)$is synchronously regular. Certainly we have

$$
L_{a} \cap\left(A^{+} \times A^{+}\right)=\bigcup_{i \in I, \lambda \in \Lambda} L_{a, i, \lambda}
$$

where each

$$
L_{a, i, \lambda}=\left\{(u, v) \in L_{a} \mid u, v \in A^{+}, u \rho \pi_{1}=v \rho \pi_{1}=i, u \rho \pi_{3}=\lambda\right\} .
$$

If $P_{\lambda,\left(a \rho \pi_{1}\right)}=0$ then $L_{a, i, \lambda}$ is empty and hence synchronously regular. Otherwise, let $w$ be a path in $K$ such that $w \sigma=P_{\lambda,\left(a \rho \pi_{1}\right)}\left(a \rho \pi_{2}\right)$. It follows from [17. Proposition 4.2] the language $K_{w}$ is regular. Now using (1) we have

$$
\begin{aligned}
L_{a, i, \lambda} & =\left\{(u, v) \in\left(K \cap X_{i \lambda}\right) \phi_{i \lambda} \times\left(K \cap X_{i, a \rho \pi_{3}}\right) \phi_{i, a \rho \pi_{3}} \mid(u \rho)(a \rho)=v \rho\right\} \\
& =\left\{(u, v) \in\left(K \cap X_{i \lambda}\right) \phi_{i \lambda} \times\left(K \cap X_{i, a \rho \pi_{3}}\right) \phi_{i, a \rho \pi_{3}}\right. \\
& \left.\quad \mid\left(u \rho \pi_{2}\right) P_{\lambda, a \rho \pi_{1}}\left(a \rho \pi_{2}\right)=v \rho \pi_{2}\right\} \\
& =\left\{\left(x \phi_{i \lambda}, y \phi_{i, a \rho \pi_{3}}\right) \mid(x, y) \in K_{w} \cap\left(X_{i \lambda} \times X_{i, a \rho \pi_{3}}\right)\right\} .
\end{aligned}
$$


But $\phi_{i \lambda}$ and $\phi_{i, a \rho \pi_{3}}$ are strongly mutually synchronous regularity preserving, so it follows that $L_{a, i, \lambda}$ is synchronously regular. Hence, $L_{a}$ is a finite union of synchronously regular binary relations, and by Lemma 2.1(iv) is synchronously regular as required.

This result extends easily from small categories to semigroupoids.

Theorem 4.4. Let $M=M^{0}(S ; F(I), G(\Lambda) ; P)$ be a Rees matrix semigroup with zero over a semigroupoid $S$. Suppose $S$ is automatic and $M$ is finitely generated. Then $M$ is automatic.

Proof. Let $P^{\prime}$ be the set of non-zero entries in the sandwich matrix $P$ and let $U=S P^{\prime} S$. Since $M$ is finitely generated, Theorem 4.2 tells us that $I, \Lambda$ and $S \backslash U$ are all finite. Let $\bar{S}$ be the category obtained from $S$ by adjoining a new identity arrow $1_{e}$ at every object $e \in S^{0}$ (even if there is already an identity arrow at $e$ ). Since $I$ and $\Lambda$ are finite, the images $I F$ and $\Lambda G$ of $F$ and $G$ are finite. But since $S$ is isolation-free, it follows from Proposition 4.1 that $S^{0}$ is the union of $I F$ and $G \Lambda$, so $S$ has only finitely many objects. It follows that $S$ is a cofinite subsemigroupoid of $\bar{S}$ so, by Theorem [2.5] $\bar{S}$ is automatic.

Define $\bar{I}=I \cup\left\{i_{s} \mid s \in S^{0} \backslash I F\right\}$ and extend $F$ to a function $\bar{F}: \bar{I} \rightarrow S^{0}$ by defining $i_{s} \bar{F}=s$ for all $s \in S^{0} \backslash I F$. Similarly, define $\bar{\Lambda}=\Lambda \cup\left\{\lambda_{s} \mid s \in\right.$ $\left.S^{0} \backslash \Lambda G\right\}$ and extend $G$ to a function $\bar{G}: \bar{\Lambda} \rightarrow S^{0}$ by defining $\lambda_{s} \bar{G}=s$ for all $s \in S^{0} \backslash \Lambda G$. Extend $P$ to a $\bar{\Lambda} \times \bar{I}$ matrix $\bar{P}$, by defining all new entries to be zero.

Now let $\bar{M}=M^{0}(\bar{S} ; \bar{F}(\bar{I}), \bar{G}(\bar{\Lambda}) ; \bar{P})$. We know that $\bar{I}$ and $\bar{\Lambda}$ are finite and that $\bar{S}$ is automatic. Furthermore, the set of non-zero entries in $\bar{P}$ is exactly the set $P^{\prime}$ of non-zero entries in $P$. Now if we let $\bar{U}=\bar{S} P^{\prime} \bar{S}$, then we have $U \subseteq \bar{U}$, from which it follows that $\bar{S} \backslash \bar{U}$ is finite. It follows by Theorem 4.3 that $\bar{M}$ is automatic.

Now clearly every non-zero element of $M$ is also contained in $\bar{M}$. Moreover, the multiplication in $M$ is clearly the same as that in $\bar{M}$, so we conclude that $M$ is a subsemigroup of $\bar{M}$. Furthermore, the only elements of $\bar{M}$ not in $M$ are those of the form $\left(i, 1_{e}, \lambda\right)$ for $i \in \bar{I}, \lambda \in \bar{\Lambda}$ and $e \in S^{0}$ with

$$
i \bar{F}=1_{e} \alpha=e=1_{e} \omega=\lambda \bar{G} .
$$

Since $S$ has finitely many objects and $\bar{I}$ and $\bar{\Lambda}$ are finite it follows that $M$ is a cofinite subsemigroup of $\bar{M}$. By Theorem [2.5 (or [11, Theorem 1.1]), it follows that $M$ is automatic.

Combining Theorem 4.4 with Theorem 4.2 we obtain a more explicit sufficient condition, without reference to $M$ being finitely generated.

Corollary 4.5. Let $M=M^{0}(S ; F(I), G(\Lambda) ; P)$ be a Rees matrix semigroup with zero over a semigroupoid $S$. If

(i) the indexing sets $I$ and $\Lambda$ are finite;

(ii) the semigroupoid $S$ is automatic; and

(iii) $S \backslash S P^{\prime} S$ is finite, where $P^{\prime}$ is the set of non-zero entries in the sandwich matrix $P$

then $M$ is automatic. 
Next, we give a sufficient condition for the underlying semigroupoid of an automatic Rees matrix semigroup to be automatic.

Let $M=M^{0}(S ; F(I), G(\Lambda) ; P)$ be a Rees matrix semigroup with zero over a semigroupoid $S$. Let $T$ be a subset of $S$. We say that $T$ is strongly right-ideal-generated by a row cross-section of $P$ if there exists a subset $\Lambda^{\prime} \subseteq \Lambda$ such that the restriction of $G$ to $\Lambda^{\prime}$ is bijective, and every arrow in $T$ can be written in the form $P_{\lambda i} s$ for some $\lambda \in \Lambda^{\prime}, i \in I$ and $s \in S^{1}$. We say that $T$ is (weakly) right-ideal-generated by a row cross-section of $P$ if there exists $\Lambda^{\prime}$ as above, such that every arrow in $T$ can be written either in the form $P_{\lambda i} s$ or in the form $P_{\lambda i}$ (or both) for some $\lambda \in \Lambda^{\prime}, i \in I$ and (where appropriate) $s \in S^{1}$.

The next result says that the set of non-identity elements of $S$ being strongly right-ideal-generated by a row cross-section of $P$ is a sufficient condition for automaticity in $M$ to imply automaticity in $S$. We shall subsequently strengthen the result by weakening the hypothesis, showing that it suffices for the set of non-identity elements of $S$ to be weakly right-idealgenerated by a row cross-section of $P$.

Theorem 4.6. Let $M=M^{0}(S ; F(I), G(\Lambda) ; P)$ be a Rees matrix semigroup with zero over a semigroupoid $S$. Suppose $M$ is automatic, and the set of non-identity elements of $S$ is strongly right-ideal-generated by a row crosssection of $P$. Then $S$ is automatic.

Proof. It follows from [17, Corollary 4.6] (or [3, Corollary 5.6]) that $M$ has an automatic cross-section and then from Proposition 3.2 that $M$ has an automatic cross-section $(A, L, \rho)$ with the property that the restriction of $\rho$ to $A$ is injective. Let $\Lambda^{\prime} \subseteq \Lambda$ be such that the restriction of $G$ to $\Lambda^{\prime}$ is bijective, and every non-identity arrow in $S$ can be written in the form $P_{\lambda i} S$ for some $\lambda \in \Lambda^{\prime}, i \in I$ and $s \in S^{1}$.

Choose some subset $I^{\prime} \subseteq I$ such that $F$ restricts to a bijection on $I^{\prime}$. Let $F^{\prime}: S^{1} \alpha \rightarrow I^{\prime}$ and $G^{\prime}: S^{1} \omega \rightarrow \Lambda^{\prime}$ be the inverses of the restrictions of $F$ and $G$ to $I^{\prime}$ and $\Lambda^{\prime}$ respectively. We define sets

$$
C=\left\{c_{s} \mid s \in S,(i, s, \lambda) \in A \rho \text { for some } i \in I, \lambda \in \Lambda\right\}
$$

and

$$
D=\left\{d_{\lambda i} \mid \lambda \in \Lambda, i \in I, P_{\lambda i} \neq 0\right\} .
$$

Clearly, $C$ and $D$ are finite. Let $X$ be a graph with vertex set $S^{0}$, and edge set $C \cup D$ where $c_{s} \alpha=s \alpha, c_{s} \omega=s \omega, d_{\lambda i} \alpha=P_{\lambda i} \alpha$ and $d_{\lambda i} \omega=P_{\lambda i} \omega$. Then there is a natural morphism $\sigma: X^{+} \rightarrow S$, given by $c_{s} \sigma=s$ and $d_{\lambda i} \sigma=P_{\lambda i}$. Note that $w \sigma \alpha=w \alpha$ and $w \sigma \omega=w \omega$ for every $w \in X^{1}$ and hence for every $w \in X^{+}$.

Let $V$ be the language

$$
V=\left(I^{\prime} \times S \times \Lambda^{\prime}\right) \rho^{-1}
$$

of words in $A^{+}$which represent non-zero elements of the form $(i, s, \lambda) \in M$ with $i \in I^{\prime}$ and $\lambda \in \Lambda^{\prime}$. Define a function $\phi: V \rightarrow X^{+}$by

$$
\left(a_{1} a_{2} \ldots a_{n}\right) \phi=c_{s_{1}} d_{\lambda_{1} i_{2}} c_{s_{2}} d_{\lambda_{2} i_{3}} \ldots d_{\lambda_{n-1} i_{n}} c_{s_{n}}
$$

where each $a_{k} \rho=\left(i_{k}, s_{k}, \lambda_{k}\right)$. That $\phi$ is a well-defined function into $X^{+}$ follows from the fact that words in $V$ represent non-zero elements of $M$. 
Furthermore, if

$$
\left(a_{1} a_{2} \ldots a_{n}\right) \phi=c_{s_{1}} d_{\lambda_{1} i_{2}} c_{s_{2}} d_{\lambda_{2} i_{3}} \ldots d_{\lambda_{m-1} i_{m}} c_{s_{m}}=\left(b_{1} b_{2} \ldots b_{n^{\prime}}\right) \phi
$$

then clearly $n=m=n^{\prime}$ and for $1 \leq k \leq n$ we have

$$
a_{k} \rho=\left(i_{k}, s_{k}, \lambda_{k}\right)=b_{k} \rho
$$

where $i_{1}=s_{1} \alpha F^{\prime}$ and $\lambda_{m}=s_{m} \omega G^{\prime}$. But $\rho$ is by assumption injective when restricted to the alphabet $A$, so it follows that $\phi$ is injective.

Notice also that for any $w \in V$ we have

$$
w \rho=\left(w \phi \sigma \alpha F^{\prime}, w \phi \sigma, w \phi \sigma \omega G^{\prime}\right) .
$$

Our aim is to show that the function $\phi$ has a regular image and a selfsynchronised sliding window inverse. We claim first that the image $V \phi$ of the function $\phi$ is regular. We say that an ordered triple $\left(d_{\lambda i}, c_{s}, d_{\mu j}\right) \in D \times C \times D$ is a valid internal triple if there exists a letter $a \in A$ with $a \rho=(i, s, \mu)$. We say that $\left(c_{s}, d_{\lambda i}\right) \in C \times D$ is a valid start pair if there exists $a \in A$ with $a \rho=\left(i^{\prime}, s, \lambda\right)$ for some $i^{\prime} \in I^{\prime}$. We say that $\left(d_{\lambda i}, c_{s}\right) \in D \times C$ is a valid end pair if there exists $a \in A$ with $a \rho=\left(i, s, \lambda^{\prime}\right)$ for some $\lambda^{\prime} \in \Lambda^{\prime}$. Now it is easily verified that

$$
V \phi \cap X^{\geq 5}=Q_{1}(C D)^{*} C Q_{2} \backslash(C \cup D)^{*} Q_{3}^{\prime}(C \cup D)^{*}
$$

where

$$
\begin{gathered}
Q_{1}=\{c d \in C D \mid(c, d) \text { is a valid start pair }\} \\
Q_{2}=\{d c \in D C \mid(d, c) \text { is a valid end pair }\}, \text { and } \\
Q_{3}^{\prime}=\{c d e \in D C D \mid(c, d, e) \text { is not a valid internal triple }\}
\end{gathered}
$$

are all finite. It follows that $V \phi \cap X^{\geq 5}$ is regular, and hence that $V \phi$ is regular, as required.

Next, we claim that $\phi$ has a self-synchronised sliding window inverse. Consider a three-letter prefix $v_{1} v_{2} v_{3}$ of a word

$$
\left(a_{1} a_{2} \ldots a_{n}\right) \phi \in V \phi \text {. }
$$

We shall define

$$
\left(v_{1} v_{2} v_{3}\right) f=a_{1} \text {. }
$$

To show that $f$ is well-defined, suppose $\left(b_{1} b_{2} \ldots b_{m}\right) \phi$ also has the prefix $v_{1} v_{2} v_{3}$. Then by the definition of $\phi$ we have

$$
\left(a_{1} a_{2} \ldots a_{n}\right) \phi=c_{s_{1}} d_{\lambda_{1} i_{2}} c_{s_{2}} d_{\lambda_{2} i_{3}} \ldots d_{\lambda_{n-1} i_{n}} c_{s_{n}}
$$

where each $a_{k} \rho=\left(i_{k}, s_{k}, \lambda_{k}\right)$, and similarly

$$
\left(b_{1} b_{2} \ldots b_{m}\right) \phi=c_{t_{1}} d_{\mu_{1} j_{2}} c_{t_{2}} d_{\mu_{2} j_{3}} \ldots \ldots . d_{\mu_{m-1} j_{m}} c_{t_{m}}
$$

where each $b_{k} \rho=\left(j_{k}, t_{k}, \mu_{k}\right)$. But now we have $c_{s_{1}}=v_{1}=c_{t_{1}}$ and $d_{\lambda_{1} i_{2}}=$ $v_{2}=d_{\mu_{1} j_{2}}$. But by the definitions of $C$ and $D$, it follows that $s_{1}=t_{1}$ and $\lambda_{1}=\mu_{1}$. Furthermore, we must have $i_{1} F=s_{1} \alpha=t_{1} \alpha=j_{1} F$, but $i_{1}, j_{1} \in I^{\prime}$ and $F$ is injective on $I^{\prime}$, so we must have $i_{1}=j_{1}$. Thus, we obtain

$$
a_{1} \rho=\left(i_{1}, s_{1}, \lambda_{1}\right)=\left(j_{1}, t_{1}, \mu_{1}\right)=b_{1} \rho .
$$

But $\rho$ is, by assumption, injective on the alphabet $A$, so we must have $a_{1}=b_{1}$, as required to show that $f$ is well-defined.

Similarly, given a word

$$
\left(a_{1} a_{2} \ldots a_{n}\right) \phi=c_{s_{1}} d_{\lambda_{1} i_{2}} c_{s_{2}} d_{\lambda_{2} i_{3}} \ldots d_{\lambda_{n-1} i_{n}} c_{s_{n}}
$$


we define

$$
\left(d_{\lambda_{p-1} i_{p}} c_{s_{p}} d_{\lambda_{p} i_{p+1}}\right) g=a_{p} \text { and }\left(c_{s_{p}} d_{\lambda_{p} i_{p+1}} c_{s_{p+1}}\right) g=\epsilon
$$

where $\epsilon$ denotes the empty word in $A^{*}$. A similar argument to that for $f$ shows that $g$ is well-defined.

Finally, given a three-letter suffix $v_{1} v_{2} v_{3}$ of a word $\left(a_{1} a_{2} \ldots a_{n}\right) \phi \in V \phi$, we shall define $\left(v_{1} v_{2} v_{3}\right) h=a_{n}$. Once again, a similar argument to that for $f$ shows that $h$ is well-defined.

Now suppose $w=a_{1} \ldots a_{n} \in V$ and $w \phi=y_{1} \ldots y_{m}$. Then by the definition of $\phi$, we have

$$
y_{1} \ldots y_{m}=\left(a_{1} a_{2} \ldots a_{n}\right) \phi=c_{s_{1}} d_{\lambda_{1} i_{2}} c_{s_{2}} d_{\lambda_{2} i_{3}} \ldots d_{\lambda_{n-1} i_{n}} c_{s_{n}}
$$

where each $a_{k} \rho=\left(i_{k}, s_{k}, \lambda_{k}\right)$. But now by the definitions of $f, g$ and $h$, it follows that

$$
\begin{array}{r}
\left(y_{1} y_{2} y_{3}\right) f\left(y_{2} y_{3} y_{4}\right) g\left(y_{3} y_{4} y_{5}\right) g \ldots\left(y_{m-3} y_{m-2} y_{m-1}\right) g\left(y_{m-2} y_{m-1} y_{m}\right) h \\
=a_{1} a_{2} \epsilon a_{3} \epsilon \ldots \epsilon a_{n-1} a_{n}=w
\end{array}
$$

We have shown that $(3, f, g, h)$ is a sliding window inverse for $\phi$, and by Lemma 2.2 it follows that $\phi$ is strongly regularity preserving.

Furthermore, if we define a function $\psi: \mathbb{N} \rightarrow \mathbb{N}$ by

$$
n \psi= \begin{cases}1 & \text { if } n=0 \text { or } n \text { is odd } \\ 0 & \text { otherwise }\end{cases}
$$

then since factors of paths in $V \phi$ begin with a letter from $C$ exactly if they begin in an odd position, $\psi$ synchronises $(3, f, g, h)$ with itself. We have already observed that $\phi$ is injective and has a regular image. It follows by Lemma 2.3 that $\phi$ is strongly synchronous regularity preserving.

We now define $K=(L \cap V) \phi \subseteq X^{+}$, and claim that $(A, K, \sigma)$ is an automatic structure (indeed, an automatic cross-section) for $S$.

First, we show that $\sigma$ maps $K$ bijectively onto $S$. To this end, let $s \in S$. Then there is an element $m=\left(s \alpha F^{\prime}, s, s \omega G^{\prime}\right) \in M$, so there exists a word $w \in L$ representing $m$. Indeed, since $s \alpha F^{\prime} \in I^{\prime}$ and $s \omega G^{\prime} \in \Lambda^{\prime}$, we have $w \in V$, and so $w \phi \in K$. But by (2), $w \phi \sigma=w \rho \pi_{2}=s$, so $w \phi$ is a representative in $K$ for $s$.

Furthermore, if $v \phi \in(L \cap V) \phi=K$ also represents $s$, then we must have $v \rho=\left(i^{\prime}, s, \lambda^{\prime}\right)$ where $i^{\prime} \in I^{\prime}$ and $\lambda^{\prime} \in \Lambda^{\prime}$. But for $\left(i^{\prime}, s, \lambda^{\prime}\right) \in M$, we must have $i^{\prime} F=s \alpha=i F$ and $\lambda^{\prime} G=s \omega=\lambda G$. Since $F$ and $G$ are bijective when restricted to $I^{\prime}$ and $\Lambda^{\prime}$, we must have $i=i^{\prime}$ and $\lambda=\lambda^{\prime}$, and so $v \rho=w \rho$. But $v, w \in L$ and $(A, L, \rho)$ is a cross-section for $M$, so we deduce that $v=w$, and hence $v \phi=w \phi$. Thus, $(X, K, \sigma)$ is a cross-section for $S$.

We know that $L$ is a regular language, and that $\phi$ is strongly regularity preserving, so it is immediate that $K=(L \cap V) \phi$ is a regular language. Since $(X, K, \sigma)$ is a cross-section for $S$, it follows that the binary relation

$$
K_{=}=\{(u, v) \in K \times K \mid u \sigma=v \sigma\}=\{(u, u) \mid u \in K\}
$$

is synchronously regular by Lemma 2.1(v).

Now let $b$ be an edge in $X$. We must show that

$$
K_{b}=\{(u, v) \in K \times K \mid u \omega=b \alpha,(u b) \sigma=v \sigma\}
$$


is synchronously regular.

If $b \sigma$ is an identity arrow, then it follows easily from the fact that $K_{=}$is synchronously regular that $K_{b}$ is synchronously regular, and we are done.

Otherwise, $b \sigma$ is not an identity arrow in $S$. Now by assumption, we can write $b \sigma=P_{\lambda i} c$ for some $\lambda \in \Lambda^{\prime}, i \in I$ and $c \in S^{1}$. Furthermore, we must have $b \alpha=b \sigma \alpha=\left(P_{\lambda i} c\right) \alpha=\lambda G$, so that $\lambda=b \alpha G^{\prime}$. Let $\mu=b \omega G^{\prime}$. Now $M$ has an element $(i, c, \mu)$. It follows from [17, Proposition 4.2] that the language

$$
L_{(i, c, \mu)}=\{(u, v) \in L \times L \mid(u \rho)(i, c, \mu)=v \rho\}
$$

is synchronously regular. We claim that

$$
K_{b}=\left\{(u \phi, v \phi) \mid(u, v) \in L_{(i, c, \mu)} \cap(V \times V), u \rho \pi_{3}=\lambda\right\} .
$$

It will then follow that

$$
K_{b}=\left\{(u \phi, v \phi) \mid(u, v) \in L_{(i, c, \mu)} \cap\left(A^{*} D \times A^{+}\right) \cap(V \times V)\right\}
$$

where $D=\left\{a \in A \mid a \rho \pi_{3}=\lambda\right\}$ is finite, so that $A^{*} D$ is regular by Lemma 2.1. By Lemma 2.1(iii) and (iv) it will follow that $L_{(i, c, \mu)} \cap\left(A^{*} D \times\right.$ $\left.A^{+}\right)$is synchronously regular. Finally, since $\phi$ is strongly synchronous regularity preserving, we shall deduce that $K_{b}$ is synchronously regular, as required.

To prove the claim, first suppose that $\left(a_{1}, a_{2}\right) \in K_{b}$. Then certainly $a_{1}, a_{2} \in K=(L \cap V) \phi$, so $a_{1}=u_{1} \phi$ and $a_{2}=u_{2} \phi$ for some $u_{1}, u_{2} \in L \cap V$. Now by (2) we have

$$
u_{k} \rho=\left(u_{k} \phi \sigma \alpha F^{\prime}, u_{k} \phi \sigma, u_{k} \phi \sigma \omega G^{\prime}\right)
$$

for $k=1$ and $k=2$. Now we have

$$
u_{1} \rho \pi_{3}=u_{1} \phi \sigma \omega G^{\prime}=a_{1} \sigma \omega G^{\prime}=a_{1} \omega G^{\prime}=b \alpha G^{\prime}=\lambda .
$$

Similarly, we obtain

$$
u_{1} \rho \pi_{1}=u_{1} \phi \sigma \alpha F^{\prime}=a_{1} \sigma \alpha F^{\prime}=a_{2} \sigma \alpha F^{\prime}=u_{2} \phi \sigma \alpha F^{\prime}=u_{2} \rho \pi_{1}
$$

and

$$
u_{2} \rho \pi_{3}=u_{2} \phi \sigma \omega G^{\prime}=a_{2} \sigma \omega G^{\prime}=b \sigma \omega G^{\prime}=\mu=(i, c, \mu) \pi_{3} .
$$

Finally, we have

$$
u_{2} \rho \pi_{2}=u_{2} \phi \sigma=a_{2} \sigma=\left(a_{1} \sigma\right)(b \sigma)=(a \sigma) P_{\lambda i} c=\left(\left(u_{1} \rho\right)(i, c, \mu)\right) \pi_{2} .
$$

We have shown that $\left(u_{1} \rho\right)(i, c, \mu)$ and $u_{2} \rho$ are equal in all three components and certainly $u_{1}, u_{2} \in L \cap V$, from which it follows that $\left(u_{1}, u_{2}\right) \in L_{(i, c, \mu)}$ as required.

Conversely, suppose $\left(u_{1}, u_{2}\right) \in L_{(i, c, \mu)} \cap(V \times V)$, and that $u_{1} \rho \pi_{3}=\lambda$. Then $u_{2} \rho=\left(u_{1} \rho\right)(i, c, \mu)$, and using (2I) and equating second components, we obtain

$$
u_{2} \phi \sigma=u_{2} \rho \pi_{2}=\left(u_{1} \rho \pi_{2}\right) P_{u_{1} \rho \pi_{3}, i} c=\left(u_{1} \phi \sigma\right) P_{\lambda i} c=\left(u_{1} \phi \sigma\right)(b \sigma) .
$$

But now by the definition of $K_{b}$, it follows that $\left(u_{1} \phi, u_{2} \phi\right) \in K_{b}$. It follows, as discussed above, that $K_{b}$ is synchronously regular, as required to complete the proof.

This result extends to cover the case where $S$ is only weakly right-idealgenerated by a row cross-section of $P$. 
Theorem 4.7. Let $M=M^{0}(S ; F(I), G(\Lambda) ; P)$ be a Rees matrix semigroup with zero over a semigroupoid $S$. Suppose $M$ is automatic, and the set of non-identity elements of $S$ is weakly right-ideal-generated by a row crosssection of $P$. Then $S$ is automatic.

Proof. Since $M$ is automatic it is certainly finitely generated, so Theorem 4.2 tells us that $S$ is finitely generated and that the indexing sets $I$ and $\Lambda$ are finite. Let $\bar{S}$ be the category obtained from $S$ by adjoining a new identity arrow at every object $e \in S^{0}$ which does not already have one. Define $\bar{I}, \bar{\Lambda}$, $\bar{P}, \bar{M}$ and $\bar{U}$ just as in the proof of Theorem 4.4. again noting that $\bar{I}$ and $\bar{\Lambda}$ are finite. Still reasoning as in the proof of Theorem 4.4, we deduce that $M$ is a cofinite subsemigroupoid of $\bar{M}$. It follows by Theorem 2.5] (or [11, Theorem 1.1]) that $\bar{M}$ is automatic. We also deduce that $\bar{S} \backslash \bar{U}$ is finite.

Next, we wish to show that the set of non-identity elements of $\bar{S}$ is strongly right-ideal-generated by a row cross-section of $\bar{P}$. Since the non-identity elements of $S$ are weakly right-ideal-generated by a row cross-section of $P$, we can choose some set $\Lambda^{\prime} \subseteq \Lambda$ such that the restriction of $G$ to $\Lambda^{\prime}$ is bijective, and every non-identity element of $S$ can be written in the form $P_{\lambda i} c$ or $P_{\lambda i}$ for some $\lambda \in \Lambda^{\prime}, i \in I$ and (where appropriate) $c \in S$. Let

$$
\bar{\Lambda}^{\prime}=\Lambda^{\prime} \cup(\bar{\Lambda} \backslash \Lambda) \text {. }
$$

Now suppose $s$ is a non-identity element of $\bar{S}$. Notice that, because we have adjoined an identity only where $S$ did not already have one, every identity in $S$ remains an identity in $\bar{S}$. Thus, we can assume that $s$ is a non-identity element of $S$. Thus, there exist $\lambda \in \Lambda^{\prime}$ and $i \in I$ such that either $s=P_{\lambda i} c$ for some $c \in S \subseteq \bar{S}$, or $s=P_{\lambda i}=P_{\lambda i} e$ where $e \in \bar{S}$ is the identity at $P_{\lambda i} \omega=i F$. Thus, the set of non-identity elements of $\bar{S}$ is strongly right-ideal-generated by a row cross-section of $P$.

It now follows by Theorem 4.6 that $\bar{S}$ is automatic. But as in the proof of Theorem 4.4, we deduce that $S$ is a cofinite subsemigroupoid of $\bar{S}$. Now by Theorem 2.5. it follows that $S$ is automatic, as required.

\section{Rees Matrices and Prefix-Automaticity}

In this section, we turn our attention to prefix-automaticity. The following result, which provides a sufficient condition for a Rees matrix semigroup over a semigroupoid to be prefix-automatic, generalises [21, Theorem 7.2] in the case of prefix-automaticity. As with Theorems 4.6 and 4.7 we shall prove this result first with the hypothesis that the semigroupoid $S$ is a small category and that the non-identity elements of $S$ are strongly right-idealgenerated by a row cross-section of the sandwich matrix $P$. We shall then extend the result to the more general case in which $S$ is a semigroupoid with non-identity elements weakly right-ideal-generated by a row cross-section of $P$.

Theorem 5.1. Let $M=M^{0}(S ; F(I), G(\Lambda) ; P)$ be a finitely generated Rees matrix semigroup with zero over a small category $S$. If $S$ is prefix-automatic, and the non-identity elements of $S$ are strongly right-ideal-generated by a row cross-section of $P$, then $M$ is prefix-automatic. 
Proof. Let $\Lambda^{\prime} \subseteq \Lambda$ be such that the restriction of $G$ to $\Lambda^{\prime}$ is bijective, and every non-identity arrow in $S$ can be written in the form $P_{\lambda i} s$ for some $\lambda \in \Lambda^{\prime}, i \in I$ and $s \in S$.

We define $U$ as in the proof of Theorem 4.3 once again deducing from the fact that $M$ is finitely generated, that $I, \Lambda$ and $S \backslash U$ are all finite and that $U$ is a subsemigroupoid of $S$. It follows by Theorem [2.5] that $U$ is prefixautomatic, and by [17. Proposition 4.4] that $U$ has a prefix-closed automatic structure. Note that since $S$ is finitely generated, it has finitely many objects and hence finitely many local identities. It follows by Proposition 3.2 that $U$ has a prefix-closed automatic structure $(X, K, \sigma)$ with the property that the restriction of $\rho$ to $X$ is injective. (Note that, since we require our automatic structure to be prefix-closed, we cannot insist that it should also be a crosssection.)

We define $A, B, z, L, H, \rho$ and each $X_{i \lambda}$ and $\phi_{i \lambda}$ exactly as in the proof of Theorem 4.3. and claim now that $(A \cup B \cup\{z\}, L, \rho)$ is a prefix-automatic structure for $S$.

By exactly the same arguments as in the proof of Theorem4.3 we deduce that $(A \cup B \cup\{z\}, L, \rho)$ is a regular choice of representatives for $M$, and that $L_{a}$ is synchronously regular for all letters $a \in A \cup B \cup\{z\}$. However, since $(X, K, \sigma)$ is not here assumed to be a cross-section for $S$, we cannot deduce that $(A \cup B \cup\{z\}, L, \rho)$ is a cross-section for $M$, and we must work a little harder to show that $L=$ is regular.

By Lemma 3.3. it will suffice to show that $L=\cap\left(A^{+} \times A^{+}\right)$is synchronously regular. Now certainly

$$
L=\cap\left(A^{+} \times A^{+}\right)=\bigcup_{i \in I, \lambda \in \Lambda} L_{i, \lambda}
$$

where

$$
L_{i, \lambda}=\left\{(u, v) \in L_{=} \cap\left(A^{+} \times A^{+}\right) \mid u \rho \pi_{1}=v \rho \pi_{1}=i, u \rho \pi_{3}=v \rho \pi_{3}=\lambda\right\} .
$$

But

$$
\begin{aligned}
L_{i, \lambda} & =\left\{(u, v) \in\left(K \cap X_{i \lambda}\right) \phi_{i \lambda} \times\left(K \cap X_{i \lambda}\right) \phi_{i \lambda} \mid u \rho=v \rho\right\} \\
& =\left\{\left(x \phi_{i \lambda}, y \phi_{i \lambda}\right) \mid(x, y) \in K_{=} \cap\left(X_{i \lambda} \times X_{i \lambda}\right)\right\} .
\end{aligned}
$$

From our argument in the proof of Theorem 4.3 , we know that $\phi_{i \lambda}$ is strongly synchronous regularity preserving, and we know that $K_{=}$is synchronously regular, so it follows that each $L_{i, \lambda}$ is synchronously regular. Hence, $L=\cap$ $\left(A^{+} \times A^{+}\right)$is a finite union of synchronously regular binary relations, and by Lemma 2.1(iv) is synchronously regular as required.

Thus, we conclude that $(A \cup B \cup\{z\}, L, \rho)$ is an automatic structure for $M$. It remains only to show that the language

$$
L_{=}^{\prime}=\{(u, v) \mid u \in L, v \in \operatorname{Pref}(L), u \rho=v \rho\}
$$

is synchronously regular. First, observe that we can write

$$
\begin{aligned}
L_{=}^{\prime}= & \left(L_{=}^{\prime} \cap(B \times \operatorname{Pref}(L))\right) \cup\left(L_{=}^{\prime} \cap(L \times B)\right) \cup\left(L_{=}^{\prime} \cap(\{z\} \times \operatorname{Pref}(L))\right. \\
& \cup\left(L_{=}^{\prime} \cap(L \times\{z\})\right) \cup\left(L_{=}^{\prime} \cap\left(A^{+} \times A^{+}\right)\right) .
\end{aligned}
$$


Clearly, $z$ and letters in $B$ are unique representatives in $\operatorname{Pref}(L)$ for the respective elements they represent. Thus, we have

$$
\left.L_{=}^{\prime} \cap(B \times \operatorname{Pref}(L))\right)=L_{=}^{\prime} \cap(L \times B)=\{(b, b) \mid b \in B\}
$$

and

$$
L_{=}^{\prime} \cap(\{z\} \times \operatorname{Pref}(L))=L_{=}^{\prime} \cap(L \times\{z\})=\{(z, z)\}
$$

so that the first four components of the union are synchronously regular.

It remains to show that $L_{=}^{\prime} \cap\left(A^{+} \times A^{+}\right)$is synchronously regular. Define

$$
\bar{L}=L \cup\left\{a_{i 1_{s} 1_{s} \lambda} \mid i \in I, s \in S^{0}, \lambda \in \Lambda, i F=s=\lambda G\right\} .
$$

Clearly, since $I$ and $\Lambda$ are finite and $S$ has finitely many objects, $\bar{L} \backslash L$ is finite. It follows by Lemma 3.4 that $\{A \cup B \cup\{z\}, \bar{L}, \rho)$ is an automatic structure for $M$.

Given any letter $a=a_{i s t \lambda} \in A$, we define $\bar{a}=a_{i s 1_{s \omega} \lambda^{\prime}}$, where $\lambda^{\prime}=s \omega G^{\prime}$ is the unique element in $\Lambda^{\prime}$ satisfying $\lambda^{\prime} G=s \omega$.

Now consider a non-empty (not necessarily proper) prefix $a_{1} \ldots a_{k}$ of a word $a_{1} \ldots a_{n}$ in $L \cap A^{+}$. We claim that $a_{1} \ldots a_{k-1} \overline{a_{k}} \in \bar{L}$. If $k=1$ then $\overline{a_{k}}$ is of the form $a_{i 1_{s} 1_{s} \lambda}$, and so is by definition in $\bar{L}$. Otherwise, we observe that from the definitions of $L$ and $\phi_{i \lambda}$ we must have

$$
a_{1} \ldots a_{n}=\left(y_{1} \ldots y_{n-1}\right) \phi_{i \lambda}
$$

for some $i \in I, \lambda \in \Lambda$ and some word $y_{1} \ldots y_{n-1} \in K \cap X_{i \lambda}$. But $K$ is prefix-closed, so also $y_{1} \ldots y_{k-1} \in K$. Furthermore, if we let $\mu \in \bar{\Lambda}$ with $\mu G=y_{k-1} \omega$ then we have $y_{1} \ldots y_{k-1} \in K \cap X_{i \mu}$ and

$$
\left(y_{1} \ldots y_{k-1}\right) \phi_{i \mu}=a_{1} \ldots a_{k-1} \overline{a_{k}} \text {. }
$$

Thus, $a_{1} \ldots a_{k-1} \overline{a_{k}} \in L \subseteq \bar{L}$ as claimed.

Recall from page 10 the definitions of the subset $H \subseteq S$ and the alphabet $A$. Let $s \in H$ and $\lambda \in \Lambda$ be such that $s \omega=\lambda F$. We consider separately the cases in which $s$ is and is not an identity element in $S$.

First, suppose that $s$ is not an identity element. Then by assumption, we can choose $\lambda^{\prime} \in \Lambda^{\prime}, i_{s} \in I$ and $t_{s} \in S$ such that $s=P_{\lambda^{\prime} i_{s}} t_{s}$. Then certainly $i_{s} F=t_{s} \alpha$ and $t_{s} \omega=s \omega=\lambda F$, so there exists an element $\left(i_{s}, t_{s}, \lambda\right) \in M$.

Since we have shown that $(A \cup B \cup\{z\}, \bar{L}, \rho)$ is an automatic structure for $M$, we deduce using [17, Proposition 4.2] that the language

$$
\bar{L}_{\left(i_{s}, t_{s}, \lambda\right)}=\left\{(u, v) \in \bar{L} \times \bar{L} \mid(u \rho)\left(i_{s}, t_{s}, \lambda\right)=v \rho\right\}
$$

is synchronously regular. We define a new language

$$
\begin{gathered}
N_{s, \lambda}=\left\{\left(u, a_{1} \ldots a_{k}\right) \mid u \in L, a_{1} \ldots a_{k} \in \operatorname{Pref}(L), a_{k}=a_{i, s^{\prime}, s, \lambda},\right. \\
\left.\left(a_{1} \ldots a_{k-1} \overline{a_{k}}, u\right) \in \bar{L}_{\left(i_{s}, t_{s}, \lambda\right)}\right\}
\end{gathered}
$$

It follows easily from Proposition 3.1 and Lemma 2.1 that $N_{s, \lambda}$ is synchronously regular.

Now for any $u \in L$ and $a_{1} \ldots a_{k} \in \operatorname{Pref}(L)$ with $a_{k}$ of the form $a_{i, s^{\prime}, s, \lambda}$ we have

$$
\overline{a_{k}} \rho \pi_{3}=s^{\prime} \omega G^{\prime}=s \alpha G^{\prime}=P_{\lambda^{\prime} i_{s}} \alpha G^{\prime}=\lambda^{\prime}
$$


so that

$$
\begin{aligned}
\left(u, a_{1} \ldots a_{k}\right) \in N_{s, \lambda} & \Longleftrightarrow\left(a_{1} \ldots a_{k-1} \overline{a_{k}}, u\right) \in \bar{L}_{\left(i_{s}, t_{s}, \lambda\right)} \\
& \Longleftrightarrow\left(a_{1} \ldots a_{k-1} \overline{a_{k}}\right) \rho\left(i_{s}, t_{s}, \lambda\right)=u \rho \\
& \Longleftrightarrow\left(a_{1} \ldots a_{k-1}\right) \rho\left(i, s^{\prime} P_{\lambda^{\prime} i_{s}} t_{s}, \lambda\right)=u \rho \\
& \Longleftrightarrow\left(a_{1} \ldots a_{k-1}\right) \rho\left(i, s^{\prime} s, \lambda\right)=u \rho \\
& \Longleftrightarrow\left(a_{1} \ldots a_{k}\right) \rho=u \rho \\
& \Longleftrightarrow\left(u, a_{1} \ldots a_{k}\right) \in L_{=}^{\prime} .
\end{aligned}
$$

Next, we consider the case in which $s$ is a local identity in $S$. In this case, we define

$$
\begin{aligned}
N_{s, \lambda}=\{( & \left(b_{1} \ldots b_{n}, a_{1} \ldots a_{k}\right) \mid a_{1} \ldots a_{k} \in \operatorname{Pref}(L), b_{1} \ldots b_{n} \in L \\
& \left.a_{k}=a_{i, s^{\prime}, s, \lambda}, b_{n}=a_{j, t, 1_{t \alpha}, \lambda},\left(a_{1} \ldots a_{k-1} \overline{a_{k}}, b_{1} \ldots b_{n-1} \overline{b_{n}}\right) \in \bar{L}_{=}\right\} .
\end{aligned}
$$

Since $(A \cup B \cup\{z\}, \bar{L}, \rho)$ is an automatic structure for $M$, we deduce that $\bar{L}_{=}$is regular, and then by Proposition 3.1 and Lemma 2.1(i) that $N_{s, \lambda}$ is synchronously regular.

Now for any $a=a_{1} \ldots a_{k} \in \operatorname{Pref}(L)$ and $b=b_{1} \ldots b_{n} \in L$ with $a_{k}$ of the form $a_{i, s^{\prime}, s, \lambda}$ and $b_{n}$ of the form $a_{j, t, 1_{t \alpha}, \lambda}$ we have

$$
\begin{aligned}
(b, a) \in N_{s, \lambda} & \Longleftrightarrow\left(a_{1} \ldots a_{k-1} \overline{a_{k}}, b_{1} \ldots b_{n-1} \overline{b_{n}}\right) \in \bar{L}= \\
& \Longleftrightarrow\left(a_{1} \ldots a_{k-1} \overline{a_{k}}\right) \rho=\left(b_{1} \ldots b_{n-1} \overline{b_{n}}\right) \rho \\
& \Longleftrightarrow\left(a_{1} \ldots a_{k-1}\right) \rho\left(i, s^{\prime}, \lambda G G^{\prime}\right)=\left(b_{1} \ldots b_{n-1}\right) \rho\left(j, t, \lambda G G^{\prime}\right) \\
& \Longleftrightarrow\left(a_{1} \ldots a_{k-1}\right) \rho\left(i, s^{\prime} s, \lambda\right)=\left(b_{1} \ldots b_{n-1}\right) \rho\left(j, t 1_{t \alpha}, \lambda\right) \\
& \Longleftrightarrow\left(a_{1} \ldots a_{k}\right) \rho=\left(b_{1} \ldots b_{n}\right) \rho \\
& \Longleftrightarrow\left(b_{1} \ldots b_{n}, a_{1} \ldots a_{k}\right) \in L_{=}^{\prime} .
\end{aligned}
$$

It follows from the two cases considered that

$$
L_{=}^{\prime}=\bigcup_{s \in H, \lambda \in \Lambda} N_{s, \lambda}
$$

so that $L_{=}^{\prime}$ is a union of finitely many synchronously regular languages, and hence by Lemma 2.1(iv) is synchronously regular.

We now extend this result as described above.

Theorem 5.2. Let $M=M^{0}(S ; F(I), G(\Lambda) ; P)$ be a finitely generated Rees matrix semigroup with zero over a semigroupoid $S$. If $S$ is prefix-automatic, and the set of non-identity elements in $S$ is weakly right-ideal-generated by a row cross-section of $P$, then $M$ is prefix-automatic.

Proof. We combine the methods used to prove Theorems 4.4 and 4.7.

As in the proof of Theorem 4.4, we deduce that the indexing sets $I$ and $\Lambda$ are finite. We define $\bar{S}, \bar{I}, \bar{\Lambda}, \bar{P}, \bar{M}$ and $\bar{U}$ as in the proof of Theorem 4.7. noting that $\bar{S}$ has an adjoined identity only where there was not already an identity in $S$.

As in Theorem 4.7. we deduce that $S$ is a cofinite subsemigroupoid of $\bar{S}$, that $M$ is a cofinite subsemigroup of $\bar{M}$, and that the non-identity elements of $\bar{S}$ are strongly right-ideal-generated by a row cross-section of $\bar{P}$. 
It follows by Theorem 2.5 that $\bar{S}$ is prefix-automatic, by Theorem 5.1 that $\bar{M}$ is prefix-automatic and then by Theorem 2.5 that $M$ is automatic, as required.

We now show that prefix-automaticity in a Rees matrix semigroup is a sufficient condition for prefix-automaticity in the underlying semigroupoid. The following result generalises [5], Theorem 4.2].

Theorem 5.3. Let $M=M^{0}(S ; F(I), G(\Lambda) ; P)$ be a Rees matrix semigroup with zero over a semigroupoid $S$. If $M$ is prefix-automatic then $S$ is prefixautomatic.

Proof. Suppose $M$ is prefix-automatic. Then by [17, Proposition 4.4] $M$ has a prefix-closed automatic structure, and by Proposition $3.2 M$ has a prefixclosed automatic structure $(A, L, \rho)$ with the property that the restriction of $\rho$ to $A$ is injective. (Once again, we note that, because we require an automatic structure which is prefix-closed, we cannot ask also that it be a cross-section.)

Choose some subsets $I^{\prime} \subseteq I$ and $\Lambda^{\prime} \subseteq \Lambda$ such that $F$ and $G$ restrict to bijections on $I^{\prime}$ and $\Lambda^{\prime}$ respectively. Define $F^{\prime}, G^{\prime}, C, D, X, \sigma, V, \phi$ and $K$ exactly as in the proof of Theorem 4.6. Reasoning as before, we deduce that $\phi$ is strongly regularity preserving and strongly synchronous regularity preserving, and that for any $w \in V$ we have

$$
w \rho=\left(w \phi \sigma \alpha F^{\prime}, w \phi \sigma, w \phi \sigma \omega G^{\prime}\right) .
$$

We deduce also that $(X, K, \sigma)$ is a regular choice of representatives for $S$, although we can no longer conclude that it is a cross-section. We claim that $(X, K, \sigma)$ is a prefix-automatic structure for $S$.

Because of the limited role played by the alphabet in the definition of an automatic structure, we can assume without loss of generality that for every letter $a \in A$, there is a letter in $A$ representing the element

$$
\left(a \rho \pi_{1}, a \rho \pi_{2}, a \rho \pi_{3} G G^{\prime}\right) \in M .
$$

For each $a \in A$, let $\bar{a} \in A$ be such a letter.

Let $c \in X^{1} \cap X^{0}$ be an edge or a path of length 0 in $X$. Consider the binary relation

$$
K_{c}^{\prime}=\{(x, y) \in \operatorname{Pref}(K) \times K \mid x \omega=c \alpha,(x c) \sigma=y \sigma\} .
$$

Our aim is to show that $K_{c}^{\prime}$ is synchronously regular. We claim that

$$
K_{c}^{\prime}=\left(\bigcup_{\lambda \in \Lambda, i \in I, b \in C \cup C D} K_{\lambda, i, b}\right) \cup\left(\bigcup_{x \in \operatorname{Pref}(K) \cap X \leq 4} K_{c}^{\prime} \cap(\{x\} \times K)\right)
$$

where each

$$
\begin{aligned}
K_{\lambda, i, b}=\{ & \left(\left(\left(w_{1} \ldots w_{n-1} \overline{w_{n}}\right) \phi\right) d_{\lambda i} b, v \phi\right) \mid w_{1}, w_{2}, \ldots, w_{n} \in A, v \in L \cap V \\
& \left.w=w_{1} \ldots w_{n} \in L, w \rho \pi_{3}=\lambda,\left(w_{1} \ldots w_{n}, v\right) \in L_{\left(i,(b c) \sigma, c \omega G^{\prime}\right)}\right\} .
\end{aligned}
$$

To prove the claim, suppose first that $(x, y) \in K_{c}^{\prime}$. Clearly, if $|x| \leq 4$ then, since $(x, y) \in K_{c}^{\prime} \cap(\{x\} \times K)$, we see that $(x, y)$ is contained in the righthand-side of (4) and we are done. 
Now suppose $|x| \geq 5$. Certainly $y \in K=(L \cap V) \phi$, so we can write $y=v \phi$ for some $v \in L \cap V$. Also $x \in \operatorname{Pref}(K)=\operatorname{Pref}((L \cap V) \phi)$, so certainly we can choose $w \in L \cap V$ and $z \in X^{*}$ with $x z=w \phi$. From the definition of $\phi$, we have $x z \in C(D C)^{*}$. Since $|x| \geq 5$, it follows that we can write $x=a d_{\lambda i} b$ for some $a \in C D C(D C)^{*}, \lambda \in \Lambda, i \in I$ and either $b \in C$ or $b \in C D$.

Suppose $w=w_{1} \ldots w_{k}$. Then it is easily verified from the definition of $\phi$ (see page 15) that $a=\left(w_{1} \ldots w_{n-1} \overline{w_{n}}\right) \phi$ where $n=(|a|+1) / 2$. Let $u=w_{1} \ldots w_{n}$, noticing that we have $u \rho \pi_{3}=\lambda$. Now we have

$$
\begin{aligned}
(u \rho)\left(i,(b c) \sigma, c \omega G^{\prime}\right) & =\left(u \rho \pi_{1}, u \rho \pi_{2}, \lambda\right)\left(i,(b c) \sigma, c \omega G^{\prime}\right) \\
& =\left(u \rho \pi_{1}, a \sigma, \lambda\right)\left(i,(b c) \sigma, c \omega G^{\prime}\right) \\
& =\left(u \rho \pi_{1},(a \sigma) P_{\lambda i}(b c) \sigma, c \omega G^{\prime}\right) \\
& =\left(u \rho \pi_{1},\left(a d_{\lambda i} b c\right) \sigma, c \omega G^{\prime}\right) \\
& =\left(v \rho \pi_{1},(x c) \sigma, v \rho \pi_{3}\right) \\
& =\left(v \rho \pi_{1}, y \sigma, v \rho \pi_{3}\right) \\
& =v \rho
\end{aligned}
$$

so that $(u, v) \in L_{\left(i,(b c) \sigma, c \omega G^{\prime}\right)}$ and hence $\left(\left(\left(w_{1} \ldots w_{n-1} \overline{w_{n}}\right) \phi\right) d_{\lambda i} b, v \phi\right) \in$ $K_{\lambda, i, b}$.

Conversely, suppose $w=w_{1} \ldots w_{n} \in L$ with

$$
\left.\left(\left(w_{1} \ldots w_{n-1} \overline{w_{n}}\right) \phi\right) d_{\lambda i} b, v \phi\right) \in K_{\lambda, i, b}
$$

for some $\lambda \in \Lambda, i \in I$ and $b \in C \cup C D$. Let $a=\left(w_{1} \ldots w_{n-1} \overline{w_{n}}\right) \phi$. Now

$$
\begin{aligned}
\left(v \rho \pi_{1},\left(\left(\left(w_{1} \ldots w_{n-1} \overline{w_{n}}\right) \phi\right) d_{\lambda i} b c\right) \sigma, v \rho \pi_{3}\right) & =\left(w \rho \pi_{1},\left(a d_{\lambda i} b c\right) \sigma, c \omega G^{\prime}\right) \\
& =\left(w \rho \pi_{1},(a \sigma) P_{\lambda i}(b c) \sigma, c \omega G^{\prime}\right) \\
& =\left(w \rho \pi_{1}, a \sigma, \lambda\right)\left(i,(b c) \sigma, c \omega G^{\prime}\right) \\
& =\left(w \rho \pi_{1}, w \rho \pi_{2}, \lambda\right)\left(i,(b c) \sigma, c \omega G^{\prime}\right) \\
& =(w \rho)\left(i,(b c \sigma), c \omega G^{\prime}\right) \\
& =v \rho
\end{aligned}
$$

so in particular we have

$$
\left(\left(\left(w_{1} \ldots w_{n-1} \overline{w_{n}}\right) \phi\right) d_{\lambda i} b \sigma\right)(c \sigma)=v \phi \sigma
$$

so that $\left(\left(\left(w_{1} \ldots w_{n-1} \overline{w_{n}}\right) \phi\right) d_{\lambda i} b \sigma, v \phi \sigma\right) \in K_{c}^{\prime}$ as required.

Also, it is clear that if $x \in \operatorname{Pref}(K) \cap X^{\leq 4}$ and $y \in K$ are such that $(x, y) \in K_{c}^{\prime} \cap(\{x\} \times K)$ then we must have $(x, y) \in K_{c}^{\prime}$. Thus, we have justified our claim that (4) holds.

Now using [17, Proposition 4.2] we see that each binary relation of the form $L_{\left(i,(b c) \sigma, c \omega G^{\prime}\right)}$ is synchronously regular. Furthermore, the functions given by

$$
\begin{gathered}
A \rightarrow A, a \mapsto \bar{a}, \text { and } \\
X \rightarrow X^{|b|+2}, x \mapsto x d_{\lambda i} b
\end{gathered}
$$

both satisfy the conditions of Proposition 3.1. Since $\phi$ is also synchronous regularity preserving, it follows that each $K_{\lambda, i, b}$ is synchronously regular.

Now let $x \in \operatorname{Pref}(K) \cap X^{\leq 4}$. Choose a word $u$ in $L$ representing the element $\left(x \alpha F^{\prime},(x \sigma)(c \sigma), c \omega G^{\prime}\right)$. Now for any word $v \phi \in(L \cap V) \phi=K$, we 
have $(x c) \sigma=v \phi \sigma$ if and only if $u \rho=v \rho$, which in turn is true exactly if $(u, v) \in L_{=}$. Thus, we have

$$
K_{c}^{\prime} \cap(\{x\} \times K)=\{x\} \times\left((\{u\} \times L) \cap L_{=}\right) \pi_{2} \phi .
$$

We know that $L$ is regular and that $L_{=}$is synchronously regular, so it follows by Lemma 2.1(ii), (iii) and (iv) and the fact that $\phi$ is strongly synchronous regularity preserving that $K_{c}^{\prime} \cap(\{x\} \times K)$ is synchronously regular.

We have shown that each $K_{c}^{\prime}$ is a finite union of synchronously regular binary relations, and it follows by Lemma2.1(iv) that each $K_{c}^{\prime}$ is synchronously regular. Now for every $c \in X^{1} \cup X^{0}$ we see that $K_{c}=K_{c}^{\prime} \cap(K \times K)$ is synchronously regular by Lemma 2.1(iv), so that $(X, L, \sigma)$ is an automatic structure for $S$. Moreover,

$$
K_{=}^{\prime}=\bigcup_{c \in X^{0}}\left(K_{c}^{\prime}\right)^{-1}
$$

is also synchronously regular by Lemma 2.1(iv), so that $(X, K, \sigma)$ is a prefixautomatic structure for $S$, as required.

\section{Rees Matrix Semigroups Without Zero}

Theorem 2.5 ensures that our results about Rees matrix semigroups with zero adapt easily to the case of Rees matrix semigroups without zero.

Theorem 6.1. Let $M=M(S ; F(I), G(\Lambda) ; P)$ be a Rees matrix semigroup (without zero) over a semigroupoid $S$. Suppose $S$ is automatic and $M$ is finitely generated. Then $M$ is automatic.

Proof. Consider the Rees matrix semigroup with zero

$$
M^{\prime}=M^{0}(S ; F(I), G(\Lambda) ; P) .
$$

Then $M=M^{\prime} \backslash\{0\}$ is a cofinite subsemigroup of $M^{\prime}$. So by [20, Theorem 1.1], $M^{\prime}$ is finitely generated. Now by Theorem 4.4. $M^{\prime}$ is automatic, and by Theorem 2.5 (or [11, Theorem 1.1]) it follows that $M$ is automatic.

Theorem 6.2. Let $M=M(S ; F(I), G(\Lambda) ; P)$ be a Rees matrix semigroup (without zero) over a semigroupoid $S$. Suppose the set of non-identity elements of $S$ is weakly right-ideal-generated by a row cross-section of $P$. If $M$ is automatic then $S$ is automatic.

Proof. Consider the Rees matrix semigroup with zero

$$
M^{\prime}=M^{0}(S ; F(I), G(\Lambda) ; P) .
$$

Then $M=M^{\prime} \backslash\{0\}$ is a cofinite subsemigroupoid of $M^{\prime}$. Now if $M$ is automatic then by Theorem 2.5. $M^{\prime}$ is automatic, and by Theorem 4.7, $S$ is automatic.

Theorem 6.3. Let $M=M(S ; F(I), G(\Lambda) ; P)$ be a finitely generated Rees matrix semigroup (without zero) over a semigroupoid $S$. If $S$ is prefixautomatic, and the set of non-identity elements of $S$ is weakly right-idealgenerated by a row cross-section of $P$, then $M$ is prefix-automatic. 
Proof. Consider the Rees matrix semigroup with zero

$$
M^{\prime}=M^{0}(S ; F(I), G(\Lambda) ; P) .
$$

Then $M=M^{\prime} \backslash\{0\}$ is a cofinite subsemigroup of $M^{\prime}$. So by [20, Theorem 1.1], $M^{\prime}$ is finitely generated. Now by Theorem [5.2. $M^{\prime}$ is prefixautomatic, and by Theorem 2.5 it follows that $M$ is prefix-automatic.

Theorem 6.4. Let $M=M(S ; F(I), G(\Lambda) ; P)$ be a Rees matrix semigroup (without zero) over a semigroupoid $S$. If $M$ is prefix-automatic then $S$ is prefix-automatic.

Proof. Consider the Rees matrix semigroup with zero

$$
M^{\prime}=M^{0}(S ; F(I), G(\Lambda) ; P) .
$$

Then $M=M^{\prime} \backslash\{0\}$ is a cofinite subsemigroupoid of $M^{\prime}$. Now if $M$ is prefixautomatic then by Theorem 2.5, $M^{\prime}$ is prefix-automatic. By Theorem 5.3, it follows that $S$ is prefix-automatic.

\section{Closing Remarks}

The curious relationship between the results of Section 5 and those of Section 4 seems to demand comment. In the case of automaticity, showing that automaticity in a Rees matrix semigroup is a sufficient condition for automaticity in the underlying semigroupoid requires a right-ideal-generation condition, while the converse implication does not. In the case of prefixautomaticity, the situation is entirely reversed - the right-ideal-generation condition is required only to show that prefix-automaticity in a Rees matrix semigroup is a necessary condition for prefix-automaticity in the underlying semigroupoid.

In [17] we remarked that it is an open question whether every automatic semigroup is prefix-automatic. Theorem 2.4 implies that this question is no harder in the ostensibly more general semigroupoid context, that is, that every automatic semigroupoid is prefix-automatic, exactly if every automatic semigroup is prefix-automatic. However, the following questions do naturally arise.

Question 7.1. Does there exist an automatic Rees matrix semigroup over a non-automatic semigroup?

Question 7.2. Does there exist an automatic Rees matrix semigroup over a non-automatic semigroupoid?

Clearly, if every automatic semigroup (and hence every automatic semigroupoid) is prefix-automatic, then Theorem 5.3 gives a negative answer to both of these questions. However, if there are automatic semigroups (and hence semigroupoids) which are not prefix-automatic, then the answers to Questions 7.1 and 7.2 could be both positive, both negative, or negative and positive respectively. 


\section{ACKNOWLEDGEMENTS}

This paper was written while the author was at Carleton University, supported by the Leverhulme Trust. The research documented was conducted while the author was a research student at the University of York, funded by an EPSRC Doctoral Studentship. The author would like to thank John Fountain for all his advice and guidance, as well as Vicky Gould, Nik Ruškuc and the anonymous referees for many helpful comments. He would also like to thank Kirsty for all her support and encouragement.

\section{REFERENCES}

[1] S. Armstrong. Structure of concordant semigroups. J. Algebra, 118:205-260, 1986.

[2] C. M. Campbell, E. F. Robertson, N. Ruškuc, and R. M. Thomas. Direct products of automatic semigroups. J. Austral. Math. Soc., 69:19-24, 2000.

[3] C. M. Campbell, E. F. Robertson, N. Ruškuc, and R. M. Thomas. Automatic semigroups. Theor. Comp. Sci., 250:365-391, 2001.

[4] C. M. Campbell, E. F. Robertson, N. Ruškuc, and R. M. Thomas. Automatic completely-simple semigroups. Acta Math. Hungar., 95:201-215, 2002.

[5] L. Descalço and N. Ruškuc. On automatic Rees matrix semigroups. Comm. Algebra, 30:1207-1226, 2002.

[6] A. J. Duncan, E. F. Robertson, and N. Ruškuc. Automatic monoids and change of generators. Math. Proc. Camb. Phil. Soc., 127:403-409, 1999.

[7] D. B. A. Epstein et al. Word Processing in Groups. Jones and Bartlett, 1992.

[8] J. B. Fountain. Abundant semigroups. Proc. London Math. Soc., 44:103-129, 1982.

[9] M. Hoffmann. Automatic Semigroups. PhD thesis, Dept. of Mathematics and Computer Science, University of Leicester, 2000.

[10] M. Hoffmann, D. Kuske, F. Otto, and R. M. Thomas. Some relatives of automatic and hyperbolic groups. In G. M. S. Gomes, J.-E. Pin, and P. V. Silva, editors, Semigroups, Algorithms, Automata and Languages, 2003.

[11] M. Hoffmann, N. Ruškuc, and R. M. Thomas. Automatic semigroups with subsemigroups of finite Rees index. Int. J. Algebra Comput., 12:463-476, 2002.

[12] M. Hoffmann and R. M. Thomas. Automaticity and commutative semigroups. Glasgow Math. J., 44:167-176, 2002.

[13] M. Hoffmann and R. M. Thomas. Notions of automaticity in semigroups. Semigroup Forum, 66:337-361, 2003.

[14] J. M. Howie. Fundamentals of Semigroup Theory. Clarendon Press, 1995.

[15] J. F. P. Hudson. Regular rewrite systems and automatic structures. In Semigroups, Automata and Languages, pages 145-152. World Scientific, 1996.

[16] M. E. Kambites. Combinatorial aspects of partial algebras. PhD thesis, Dept. of Mathematics, University of York, 2003.

[17] M. E. Kambites. Automatic semigroups and categories. preprint, 2005.

[18] M. E. Kambites. Presentations for semigroups and semigroupoids. Int. J. Algebra Comput., 15:291-308, 2005.

[19] M. V. Lawson. Rees matrix semigroups over semigroupoids and the structure of a class of abundant semigroups. Acta Sci. Math., 66:517-540, 2000.

[20] N. Ruškuc. On large subsemigroups and finiteness conditions of semigroups. Proc. London Math. Soc., 76:383-405, 1998.

[21] P. V. Silva and B. Steinberg. Extensions and submonoids of automatic monoids. Theor. Comp. Sci., 289:727-754, 2002. 